\title{
ON THE TORUS THEOREM AND ITS APPLICATIONS
}

\author{
BY \\ C. D. FEUSTEL( ${ }^{1}$ ) \\ Dedicated to Professor R. H. Fox
}

\begin{abstract}
In this paper, we prove the torus theorem and that manifolds in a certain class of 3-manifolds with toral boundary are determined by their fundamental groups alone. Both of these results were reported by $F$. Waldhausen. We also give an extension of Waldhausen's generalization of the loop theorem.
\end{abstract}

I. Introduction. The primary purpose of this paper is to prove the "Torus Theorem" reported by F. Waldhausen in [17]. This theorem relates the existence of essential maps of tori into a 3-manifold $M$ and essential embeddings of tori or annuli into $M$. The reader should note that the manifold $M$ is required to be bounded and irreducible. Originally the author thought that the torus theorem would also hold if one assumed that $M$ was "sufficiently large" but not necessarily bounded. Unfortunately this is not true as is shown by an example communicated to the author by W. Jaco. Jaco obtained his example by surgery on the space of a torus knot of genus greater than two.

The results of this paper also follow from theorems proved independently by Johanssen and by Jaco and Shalen which classify the boundary preserving maps of a torus or annulus into a sufficiently large 3-manifold, up to boundary preserving homotopy.

We obtain as corollaries to the "torus theorem" partial answers to questions of Neuwirth (question $T$ in [8]) and Papakyriakopoulos (question 3 in [9]). The "torus theorem" also relates to a question of R. H. Fox (problem 4 in [7]). In the general case a negative answer to Fox's question has been given in [4] and [13].

We also obtain a result that shows that a certain class of 3-manifolds with toral boundaries are determined by their fundamental groups alone.

In order to establish the "torus theorem", we first prove supporting theorems that have some interest in their own right.

Firstly we give an extension of the "annulus theorem" reported by

Received by the editors March 14, 1974 and, in revised form, April 18, 1975.

AMS (MOS) subject classifications (1970). Primary 55A35, 55A05; Secondary 57C35, 55A25.

Key words and phrases. Torus, annulus, essential map, essential embedding.

(1) The author is partially supported by NSF Grant GP 15357. 
Waldhausen in [17] and proved in [6]. We also give an extension of Waldhausen's "generalized loop theorem" [14].

The author acknowledges his indebtedness to F. Waldhausen who announced results in [17] that suggested the direction of this paper.

II. Notation. Throughout this paper all spaces are simplicial complexes and all maps are piecewise linear. We use regular neighborhood to mean second regular neighborhood. The closure of a set $X \subset Y$ is denoted by $\operatorname{cl}(X)$. All manifolds are compact and orientable unless they arise in a proof or we admit they are nonorientable. We denote the boundary of a manifold $M$ by $\partial M$ and $M-\partial M$ by $\operatorname{int}(M)$. A manifold $N$ is properly embedded in a manifold $M$ if $N \cap \partial M=\partial N$. If $X$ and $Y$ are connected spaces, the natural map from $\pi_{1}(X)$ into $\pi_{1}(Y)$ induced by inclusion is denoted by $\pi_{1}(X) \rightarrow \pi_{1}(Y)$. A two-sided connected surface $F$ properly embedded in a 3-manifold $M$ is incompressible (in $M$ ) if $\pi_{1}(F) \rightarrow \pi_{1}(M)$ is monic. It is a well-known corollary to the loop theorem [12] that $F$ is incompressible in $M$ if and only if, for each disk $D$ embedded in $M$ such that $D \cap F=$ $\partial D, \partial D$ is nullhomotopic on $F$. A surface embedded in $M$ is incompressible in $M$ if each of its components is incompressible.

Throughout this paper $A$ will denote an annulus and $c_{1}$ and $c_{2}$ the components of $\partial A$. An arc properly embedded in $A$ whose complement is simply connected is a spanning arc of $A$. We denote one such $\operatorname{arc}$ on $A$ by $\alpha$. Let $F$ be a surface embedded in the manifold $M$. A map $f:(A, \partial A) \rightarrow(M, F)$ is $F$-essential if

(1) $f_{*}: \pi_{1}(A) \rightarrow \pi_{1}(M)$ is monic.

(2) The $\operatorname{arc} f(\alpha)$ is not homotopic rel its endpoints to an $\operatorname{arc}$ on $F$.

If $F=\partial M$, we say that $f$ is essential. The reader should observe that the definition of $F$-essential is independent of the choice of $\alpha$.

Throughout this paper $T$ will denote a 2 -dimensional torus and $D$ will denote a 2-dimensional disk. Let $M$ be a 3-manifold and $f: T \rightarrow M$ a map. Then $f$ is essential if

(1) $f_{*}: \pi_{1}(T) \rightarrow \pi_{1}(M)$ is monic.

(2) There is an element $\sigma \in \pi_{1}(T)$ such that $f_{*}(\sigma)$ has a representative loop not freely homotopic to a loop in $\partial M$.

We define an essential map of a Klein bottle in the same way. We shall say that $f$ is $S$-essential if $f$ is essential and for each $\sigma \neq 1 \in \pi_{1}(T), f_{*}(\sigma)$ has a representative not freely homotopic to a loop in $\partial M$. If $f: T \rightarrow M$ is an essential map that is not $S$-essential, we say that $f$ is $W$-essential.

Let $F_{1}$ and $F_{2}$ be disjoint surfaces properly embedded in a 3-manifold M. Then $F_{1}$ is parallel to $F_{2}$ if there is an embedding $g: F_{1} \times[0,1] \rightarrow M$ such that

(1) $g\left(F_{1} \times\{0\}\right)=F_{1}$. 
(2) $g\left(F_{1} \times\{1\}\right)=F_{2}$.

(3) $g\left(\partial F_{1} \times[0,1]\right) \subset \partial M$.

Let $f: X \rightarrow Y$ be a map. Then we denote $\operatorname{cl}\left\{x \in X: f^{-1} f(x) \neq\{x\}\right\}$ by $S(f)$. We use the terms hierarchy and boundary incompressible as they are defined in [15] and [16]. We shall denote the Euler characteristic of a surface $F$ by $\chi(F)$. Let $M$ be a 3-manifold with incompressible boundary. Let $F$ be a twosided, connected surface properly embedded in $M$. We shall say that $F$ is good (with respect to $M$ ) if

(1) $F$ is incompressible.

(2) $F$ is boundary incompressible.

(3) $F$ does not separate $M$.

(4) A two-sided surface $F_{1}$ properly embedded in $M$ and satisfying properties (1), (2) and (3) above cannot be found so that $\chi\left(F_{1}\right)>\chi(F)$.

(5) A two-sided surface $F_{1}$ properly embedded in $M$ and satisfying properties (1), (2), (3) and (4) above cannot be found so that genus $\left(F_{1}\right)<\operatorname{genus}(F)$.

Let $f: T \rightarrow M_{1}$ be an essential map. Let $M_{j}, F_{j} \subset M_{j}, U\left(F_{j}\right)$ for $j=1$, $\ldots, n$ be a hierarchy for $M_{1}$. We suppose that

(1) $F_{j}$ is a disk if $\partial M_{j}$ is compressible for $j=1, \ldots, n$.

(2) $F_{j}$ is an essential annulus if $\partial M_{i}$ is incompressible and $M_{j}$ admits an essential embedding of an annulus for $j=1, \ldots, n$.

(3) $F_{j}$ is a good surface otherwise.

We assume that if there is a map $f_{1}$ homotopic to $f$ and a surface $F_{1}^{\prime}$ satisfying (1)-(3) above such that $f_{1}(T) \cap F_{1}^{\prime}$ is empty, $F_{1}$ has been chosen to be such a surface. More generally if there is a map $f_{1}$ homotopic to $f$ such that $f_{1}(T) \subset M_{j}$ and surface $F_{j}^{\prime}$ in $M_{j}$ satisfying (1)-(3) above such that $f_{1}(T) \cap F_{j}^{\prime}$ is empty, $F_{j}$ is such a surface for $j=1, \ldots, n$.

We shall say that a hierarchy having the properties above is special with respect to $f$.

Let $M_{1}$ be an orientable 3-manifold and $F$ a two-sided connected surface properly embedded in $M_{1}$. Then the manifold $M_{2}$ obtained by splitting $M_{1}$ along $F$ has by definition the property: $\partial M_{2}$ contains surfaces $F_{1}$ and $F_{2}$ which are copies of $F$, and identifying $F_{1}$ and $F_{2}$ gives a projection $P:\left(M_{2}, F_{1} \cup F_{2}\right) \rightarrow$ $\left(M_{1}, F\right)$.

We say that a map $f: A \rightarrow M$ is transverse with respect to a surface $F$ properly embedded in $M$ if for each $x \in f^{-1}(F)$

(1) $f^{-1} f(x)$ contains at most two points.

(2) Let $R_{1}$ be a regular neighborhood of $F$.

Let $R_{2}$ be a regular neighborhood of $x \in A$. Then $R_{2}-f^{-1}(F)$ has two components and $f$ carries these components to distinct components of $R_{1}-F$.

Let $F$ be a surface properly embedded in a 3-manifold $M$. Let $F_{1}$ and $F_{2}$ 
be disjoint surfaces embedded in $M$ so that

$$
F_{j} \cap(F \cup \partial M)=\partial F_{j} \text { for } j=1,2 .
$$

We say that $F_{1}$ and $F_{2}$ are parallel rel $F$ if the surfaces in $P^{-1}\left(F_{1} \cup F_{2}\right)$ are parallel in the manifold obtained by splitting $M$ along $F$.

Let $K$ be a knot in $S^{3}$. Let $M$ be the complement of the interior of a regular neighborhood of $K$ in $S^{3}$. Then we shall say that $M$ is the knot space of $K$.

III. $F$-essential annuli. In this section we relate the existence of $F$-essential maps and $F$-essential embeddings.

LEMMA 3.1. Let $F$ be an incompressible, boundary incompressible surface properly embedded in the 3-manifold $M$. Let $f: D \rightarrow M$ be a map such that $f^{-1}(F)$ is an arc $\beta$ in $\partial D$ and $f^{-1}(\partial M)=\partial D-\operatorname{int}(\beta)$. Then there exists a map $f^{\prime}: D \rightarrow M$ such that $f^{\prime}|\beta=f| \beta, f^{\prime}(D) \subset F$ and $f^{\prime}(\partial D-\beta) \subset \partial M$.

Proof. This lemma is a weakened form of Lemma (1.7) in [16] and its proof follows immediately from the proof of (1) in that lemma.

Proposition 3.2. Let $M$ be a 3-manifold and $F$ an incompressible surface in $M$. Let $\pi_{2}(M)=0$ and $f:(A, \partial A) \rightarrow(M, F)$ a map such that $f_{*}: \pi_{1}(A) \rightarrow$ $\pi_{1}(M)$ is monic. Then $f$ is F-essential if and only if it is not homotopic rel $\partial A$ to a map into $F$.

Proof. Clearly if $f$ is homotopic to a map into $F, f$ is not $F$-essential. Suppose $f$ is not $F$-essential. Then there is a homotopy of $f \mid \alpha$ rel $\partial \alpha$ to a map into $F$. Thus we may assume that $f(\alpha)$ lies on $F$. If we split $A$ along $\alpha$, we obtain a disk $D$ and $f$ induces a map $f_{1}:(D, \partial D) \rightarrow(M, F)$. It can be seen that if $f_{1}$ is homotopic rel $\partial D$ to a map into $F, f$ is homotopic rel $\partial A$ to a map into $F$. Since $F$ is incompressible, $f_{1}(\partial D)$ is inessential on $F$. Thus there is a map $f_{2}: D \rightarrow F$ such that $f_{2}\left|\partial D=f_{1}\right| \partial D$. Since $\pi_{2}(M)=0, f_{2}$ is homotopic to $f_{1}$ rel $\partial D$. This completes the proof of Proposition 3.2.

THEOREM 1. Let $M$ be a 3-manifold and $F \subset \partial M$ an incompressible surface in $M$. Let $f:(A, \partial A) \rightarrow(M, F)$ be an F-essential map such that $f \mid \partial A$ is an embedding. Then there is an F-essential embedding $g:(A, \partial A) \rightarrow(M, F)$ such that $f(\partial A)=g(\partial A)$.

Proof. Theorem 1 is an immediate consequence of Theorem $1^{\prime}$ in [6].

THEOREM 2. Let $M$ be a 3-manifold and $F$ a surface embedded in $\partial M$ such that $\pi_{1}(F) \rightarrow \pi_{1}(M)$ is monic. Let $f:(A, \partial A) \rightarrow(M, F)$ be an F-essential map. Then there is an F-essential embedding $g:(A, \partial A) \rightarrow(M, F)$. Furthermore if $f\left(c_{1}\right) \cap f\left(c_{2}\right)$ is empty we may assume that $g\left(c_{j}\right)$ lies in any prespecified neighborhood of $f\left(c_{j}\right)$ for $j=1,2$. 
Proof. One uses the proof of Theorem 2 or Theorem 3 in [6] to obtain an $F$-essential map $f_{1}:(A, \partial A) \rightarrow(M, F)$ such that $f_{1} \mid \partial A$ is a homeomorphism. Theorem 2 can now seem to be a consequence of Theorem 1 .

IV. A generalization of the loop theorem. In this section we concern ourselves with replacing a map $f:(A, \partial A) \rightarrow(M, F)$ by an embedding $g:(A, \partial A) \rightarrow$ $(M, F)$ so that the class of the loop $g\left(c_{1}\right)$ preserves a certain property of the loop $f\left(c_{1}\right)$. In particular if $F$ is an incompressible surface in $\partial M$ and $f\left(c_{1}\right)$ is not freely homotopic to a loop in $\partial F$, we will show that we may suppose that $g$ may be chosen so that $g\left(c_{1}\right)$ is not freely homotopic to that loop in $\partial M$ as long as $f\left(c_{1}\right)$ $\cap f\left(c_{2}\right)$ is empty. In case the component $F_{1}$ of $F$ on which $f\left(c_{1}\right)$ lies is planar and $\chi\left(F_{1}\right)=-1$, Theorem 3 shows that $g$ may be chosen so that $g\left(c_{1}\right)$ is freely homotopic to any prespecified component of $\partial F_{1}$.

In Theorem 3 below we are interested only in the conjugacy class of $\left[f\left(c_{1}\right)\right], \tau_{1}, \ldots, \tau_{n}$ so we need not concern ourselves with a basepoint for $\pi_{1}(F)$.

THEOREM 3. Let $F$ be an incompressible bounded surface embedded in $\partial M$. Let $\tau_{1}, \ldots, \tau_{n}$ be elements in $\pi_{1}(F)$ with the following properties:

(1) $\tau_{i} \neq \sigma^{k}$ for all $\sigma \in \pi_{1}(F), k \neq \pm 1$, and $i=1, \ldots, n$.

(2) $\sigma_{1} \tau_{i}^{u} \sigma_{1}^{-1} \sigma_{2} \tau_{j}^{v} \sigma_{2}^{-1} \neq \sigma_{3} \tau_{k}^{w} \sigma_{3}^{-1}$ for all integers $i, j, k, u, v$, and $w$ and all elements $\sigma_{1}, \sigma_{2}, \sigma_{3} \in \pi_{1}(F)$ such that $1 \leqslant i, j, k \leqslant n, \sigma_{1} \neq \sigma_{2}$, and $u$, $v$, and $w$ nonzero.

(3) $\tau_{i}^{u} \tau_{j}^{v} \neq \sigma \tau_{k}^{w} \sigma^{-1}$ where $\sigma \in \pi_{1}(F)$ unless $i=j=k, \sigma=1$, and $u+v=$ $w$ where $u, v$, and $w$ are not equal to zero.

Let $f:(A, \partial A) \rightarrow(M, \partial M)$ be a map such that

(1) $f_{*}: \pi_{1}(A) \rightarrow \pi_{1}(M)$ is monic.

(2) $f\left(c_{1}\right) \subset F$.

(3) $f\left(c_{1}\right) \cap f\left(c_{2}\right)$ is empty.

(4) $\left[f\left(c_{1}\right)\right]$ is not conjugate to a power of $\tau_{i}$ for $1 \leqslant i \leqslant n$.

Then we can find an embedding $g:(A, \partial A) \rightarrow(M, \partial M)$ such that

(1) $g_{*}: \pi_{1}(A) \rightarrow \pi_{1}(M)$ is monic.

(2) $g\left(c_{j}\right)$ lies in any prespecified neighborhood of $f\left(c_{j}\right)$ for $j=1,2$.

(3) $\left[g\left(c_{1}\right)\right]$ is not conjugate to a power of $\tau_{i}$ for $i=1, \ldots, n$.

Proof. The proof of Theorem 3 varies very little from the standard one involving a tower of 2-sheeted coverings. Suppose that $f_{m}:(A, \partial A) \rightarrow\left(M_{m}, \partial M_{m}\right)$ is a map such that $f_{m} *\left(\pi_{1}(A)\right)$ is not contained in any subgroup of index two of $\pi_{1}\left(M_{m}\right), q: M_{m} \rightarrow M$ is a map such that $q f_{m}=f$, and $f_{m}(A)$ is a deformation retract of $M_{m}$. The usual homology arguments show that $\partial M_{m}$ is the union of a torus $T$ and a collection of 2-spheres. Since $f_{*}$ is monic, $f(\partial A) \subset T$. Let $R$ be a regular neighborhood of $f_{m}\left(c_{1}\right)$ in $T$. Let $A_{2}$ be the closure of the component of 
$T-R$ which contains $f_{m}\left(c_{2}\right)$. Since $q f_{m}\left(c_{2}\right)$ is essential and $\partial A_{2}$ can contain at most two components, $A_{2}$ is an annulus. Let $A_{1}$ be the closure of $T-A_{2}$. Let $\lambda_{j}$ be a simple loop in $f_{m}\left(c_{j}\right)$ such that $\lambda_{j}$ represents a generator of $\pi_{1}\left(A_{j}\right)$ for $j=1$, 2. Now $\left[f_{m}\left(c_{j}\right)\right]=\left[\lambda_{j}\right]^{v} \in \pi_{1}\left(A_{j}, x\right)$ where $x \in \lambda_{j}$ and $v$ is an integer. It follows that $q f_{m}\left(c_{1}\right)=f\left(c_{1}\right)$ is homotopic to a nonzero multiple of $\left.q \lambda_{1}\right)$ in $F$ since $F$ is incompressible. Thus if $\left[f\left(c_{1}\right)\right]$ is not conjugate to a power of $\tau_{i}$, $\left[q\left(\lambda_{1}\right)\right]$ will not be conjugate to a power of $\tau_{i}$ for $i=1, \ldots, n$. Let $g_{m}: A \rightarrow$ $\partial M_{m}$ be an embedding such that $g_{m}(\partial A)=\lambda_{1} \cup \lambda_{2}$. It can now be seen that we may replace the map $q f_{m}=f$ with the map $q g_{m}$. The argument above shows that the map of $A$ into the manifold at the top of the tower may be taken to be an embedding.

It remains to be shown that we can "push this embedding down the tower." Suppose $\left(M_{m}, p_{m}\right)$ is a 2-sheeted cover of $M_{m-1}, f_{m}:(A, \partial A) \rightarrow\left(M_{m}, \partial M_{m}\right)$ is an embedding, $f_{m-1}=p_{m} f_{m}$, and $q: M_{m-1} \rightarrow M$ is a map such that $q f_{m-1}=$ $f$. We may suppose that the singular set of $f_{m-1}$ is the union of a collection of pairwise disjoint arcs and loops. Clearly the loops can be disposed of by using the standard argument. Suppose $\alpha_{1}$ and $\alpha_{2}$ are disjoint spanning arcs on $A$ and that $f_{m-1}\left(\alpha_{1}\right)=f_{m-1}\left(\alpha_{2}\right)$. Let $a_{1}$ and $a_{2}$ be the closures of the components of $c_{1}-\left(\alpha_{1} \cup \alpha_{2}\right)$. Let $x$ be the point $f\left(\partial a_{1}\right)$. Now $\left[f\left(c_{1}\right)\right]=\left[f\left(a_{1}\right)\right]\left[f\left(a_{2}\right)\right]$ in $\pi_{1}(F, x)$. If $\left[f\left(a_{1}\right)\right]$ (or $\left[f\left(a_{2}\right)\right]$ ) is not of the form $\sigma_{1} \tau_{i}^{u} \sigma_{1}^{-1}$ where $\sigma_{1} \in \pi_{1}(F, x)$ and $u$ is a nonzero integer, we can find an embedding $g_{m}: A \rightarrow M_{m}$ such that $q p_{m} g_{m}\left(c_{1}\right)=f\left(a_{1}\right)$ (or $\left.f\left(a_{2}\right)\right)$. Otherwise $\left[f\left(a_{1}\right)\right]=\sigma_{1} \tau_{i}^{u} \sigma_{1}^{-1}$ and $\left[f\left(a_{2}\right)\right]=$ $\sigma_{2} \tau_{j}^{v} \sigma_{2}^{-1}$ where $i, j, u, v$ are nonzero integers such that $1 \leqslant i, j \leqslant n$ and $\sigma_{1}, \sigma_{2} \in$ $\pi_{1}(F, x)$. Since $\tau_{i} \neq \tau_{j}$ or $\sigma_{1} \neq \sigma_{2}, \sigma_{1} \tau_{i}^{u} \sigma_{1}^{-1} \sigma_{2} \tau_{j}^{-v} \sigma_{2}^{-1} \neq \sigma_{3} \tau_{k}^{w} \sigma_{3}^{-1}$ for $\sigma_{3} \in$ $\pi_{1}(F, x), 1 \leqslant k \leqslant n$, and $w$ a nonzero integer. Thus it can be seen that the usual cutting argument will suffice.

Suppose $\alpha_{1}$ and $\alpha_{2}$ are disjoint simple arcs on $A$ such that $f_{m-1}\left(\alpha_{1}\right)=$ $f_{m-1}\left(\alpha_{2}\right)$ and $\partial \alpha_{j} \subset c_{1}$ for $j=1,2$. Then $\alpha_{1}$ and $\alpha_{2}$ cut off disks $D_{1}$ and $D_{2}$ on $A$. Let $\beta_{j}$ be the closure of $\left(\partial D_{j}\right)-\alpha_{j}$ for $j=1,2$. We observe that the loop $f_{m-1}\left(\beta_{1}\right) \cup f_{m-1}\left(\beta_{2}\right)$ is nullhomotopic on the singular disk $f_{m-1}\left(D_{1} \cup D_{2}\right)$. Thus the loop $f\left(\beta_{1}\right) \cup f\left(\beta_{2}\right)$ is nullhomotopic in $M$ and thus on $F$ since $F$ is incompressible. It follows that one could have simplified the singular set of $f \mid c_{1}$ via a homotopy or that one can use the usual cutting argument.

It can be seen that if $\alpha_{1}$ and $\alpha_{2}$ are disjoint simple arcs properly embedded in $A$ such that $\partial \alpha_{j} \subset c_{2}$ for $j=1,2$ and $f_{m-1}\left(\alpha_{1}\right)=f_{m-1}\left(\alpha_{2}\right)$, the standard cutting argument yields a map agreeing with $f$ on $c_{1}$. Theorem 3 follows.

V. Supporting lemmas. In this section we prove the lemmas which are necessary in our proof of Waldhausen's "Torus theorem". It is a consequence of Lemma 5.1 below that an essential loop $\lambda$ on a good surface $F$ embedded in a 
3-manifold $M$ is not freely homotopic to a loop in $\partial M$ unless $\lambda$ is freely homotopic in $F$ to a loop in $\partial F$ or $F$ is the planar surface with three boundary components.

LEMMA 5.1. Let $F$ be an incompressible, boundary incompressible, nonseparating surface properly embedded in $M$ such that $F$ is not the planar surface with $\chi(F)=-1$. Let $f: A \rightarrow M$ be a map such that $f\left(c_{1}\right) \subset F, f\left(c_{1}\right)$ is essential, $f\left(c_{2}\right) \subset \partial M$, and $f\left(c_{1}\right)$ is not freely homotopic in $F$ to a loop in $\partial F$. Then $F$ is not good.

Proof. After a general position argument, we may suppose that there is an annular neighborhood $A_{1}$ of $c_{1}$ in $A$ such that $f^{-1}(F) \cap A_{1}=c_{1}$ since $F$ is two-sided. We may also assume that $f^{-1}(F)$ is the union of a finite collection of disjoint simple arcs and loops properly embedded in $A$. We suppose that $f$ has been chosen so that the number of components in $f^{-1}(F)$ is minimal.

Let $\lambda$ be a simple loop in $f^{-1}(F)-c_{1}$. Suppose $\lambda$ is nullhomotopic on $A$. Since $F$ is incompressible, $f(\lambda)$ is nullhomotopic on $F$. Let $D$ be a regular neighborhood of the disk bounded by $\lambda$ in $A$. Then we can alter $f$ on $D$ to obtain a map $f_{1}: A \rightarrow M$ such that $f_{1}|A-D=f| A-D$ and $f_{1}(D) \cap F$ is empty. This contradicts our assumption of minimality on $f^{-1}(F)$.

Suppose $\lambda$ is not nullhomotopic on $A$ and $f(\lambda)$ is not freely homotopic on $F$ to a loop in $\partial F$. Let $A_{1}$ be the subannulus of $A$ bounded by $c_{2}$ and $\lambda$. Then the existence of the map $f_{1}=f \mid A_{1}: A_{1} \rightarrow M$ contradicts our minimality hypothesis since $f_{1}^{-1}(F)$ contains fewer components than $f^{-1}(F)$. Thus we may suppose that $f(\lambda)$ is freely homotopic to a loop in $\partial F$. Let $A_{1}$ be a regular neighborhood of the annulus bounded by $c_{2}$ and $\lambda$. Then we can alter $f$ on $A_{1}$ to obtain a map $f_{1}:\left(A, c_{2}\right) \rightarrow(M, \partial M)$ such that $f_{1}\left|A-A_{1}=f\right| A-A_{1}$ and $f\left(A_{1}\right)$ does not meet $F$. This contradicts our minimality hypothesis above. Thus we may suppose that $f^{-1}(F)-c_{1}$ contains no simple loops.

Suppose there is an arc $\alpha$ in $f^{-1}(F)-c_{1}$. Then we can choose $\alpha$ so that there is a disk $D$ on $A$ such that $D \cap f^{-1}(F)=\alpha$ and $\partial D=\alpha \cup\left(D \cap f^{-1}(\partial M)\right)$. It is a consequence of Lemma 3.1, that we can alter $f$ on $D$ to obtain a map $f_{1}: A \rightarrow M$ such that $f_{1}|A-D=f| A-D$ and $f_{1}(D) \subset F$. One can now modify $f_{1}$ on a regular neighborhood of $D$ to obtain a map $f_{2}$ such that the number of components in $f_{2}^{-1}(F)$ is less than the number of components in $f^{-1}(F)$. This contradicts our minimality hypothesis. Thus we may suppose that $f^{-1}(F)=c_{1}$.

It is a consequence of Theorem 3 that there is an embedding $g: A \rightarrow M$ such that

(1) $g\left(c_{1}\right) \subset F$ and $g\left(c_{2}\right) \subset \partial M$.

(2) $g^{-1}(F)=c_{1}$.

(3) $g\left(c_{1}\right)$ is not freely homotopic on $F$ to a loop in $\partial M$. 
(4) $g_{*}: \pi_{1}(A) \rightarrow \pi_{1}(M)$ is monic.

Let $R$ be a regular neighborhood of $F \cup g(A)$ and $G=\operatorname{cl}(\partial R-\partial M)$. Let $F_{1}$ be the component of $G$ parallel to $F$. Now $\chi(G)=2 * \chi(F)$. If $G-F_{1}$ is connected, genus $\left(G-F_{1}\right)<\operatorname{genus}(F)$. Otherwise let $F_{2}$ and $F_{3}$ be the components of $G$. Then $\chi\left(F_{2}\right)>\chi(F)$ and $\chi\left(F_{3}\right)>\chi(F)$. Since $F$ is incompressible it can be seen that $F_{2} \cup F_{3}$ is incompressible. Since $\left[F_{1}\right]$ is not a boundary rel $\partial M$ and $\left[G-F_{1}\right]$ is homologous to $F_{1}$ rel $\partial M,\left[G-F_{1}\right]$ is not a boundary rel $\partial M$. Let $F_{2}$ be a component of $G-F_{1}$. Then we may suppose that $F_{2}$ does not separate $M$. If $F_{2}$ is boundary incompressible, it can be seen that $F$ is not good. Otherwise there is a disk $D$ embedded in $M$ such that $D \cap F_{2}$ is an arc $\beta$ and $D \cap\left(\partial M \cup F_{2}\right)=\partial D$. Using an argument similar to the one above we can find a nonseparating, incompressible surface $F_{2}^{\prime}$ properly embedded in $M$ such that $\chi\left(F_{2}^{\prime}\right)>\chi\left(F_{2}\right)$. Since $\chi\left(F_{2}^{\prime}\right) \leqslant 1$, it can be seen that $F_{2}^{\prime}$ may be taken to be boundary incompressible and thus $F$ is not good. This completes the proof of Lemma 5.1.

Lemma 5.2 below is proved using techniques similar to those used in the proof of Lemma 5.1 above and we leave most of the details to the reader.

LEMMA 5.2. Let $F$ be a good surface properly embedded in $M$ and $R a$ regular neighborhood of $F$ in $M$. Let $\pi_{2}(M)=0$. Let $g:(A, \partial A) \rightarrow(M, \operatorname{int}(F))$ be an F-essential embedding such that

(1) $g(A)$ meets only one component of $R-F$.

(2) $g\left(c_{1}\right)$ is not freely homotopic in $F$ to a loop in $\partial F$.

(3) $g_{*}: \pi_{1}(A) \rightarrow \pi_{1}(M)$ is monic.

(4) $g^{-1}(F)=\partial A$.

Let $N$ be a regular neighborhood of $F \cup g(A)$. Let $F_{1}$ be the nonseparating component of $\mathrm{cl}(\partial N-\partial M)$ not parallel to $F$. Then $F_{1}$ is good.

Proof. We observe that $\operatorname{cl}(\partial N-\partial M)$ has at most three components and that one of them say $F_{1}$ must not separate $M$. Note that $\chi\left(F_{1}\right) \leqslant \chi(F)$. If $F_{1}$ is not incompressible there is a disk $D$ embedded in $M$ such that $D \cap F_{1}=\partial D$. Using $D$ and $F_{1}$, we can find a surface $F_{2}$ properly embedded in $M$ that does not separate $M$ so that $\chi\left(F_{2}\right)>\chi\left(F_{1}\right) \geqslant \chi(F)$. Since $\pi_{2}(M)=0, F_{2}$ cannot be a 2-sphere. As in the proof of Lemma 5.1, the existence of $F_{2}$ guarantees the existence of a good surface $F_{3}$ such that $\chi\left(F_{3}\right)>\chi(F)$. This is a contradiction. Thus $F_{1}$ is incompressible. Similar arguments show $F_{1}$ is boundary incompressible. Lemma 5.2 follows.

A connected, closed 2-manifold $K$ that is the union of a collection of annuli $A_{i}$ for $i=1, \ldots, n$ such that each component of $A_{i} \cap A_{j}$ is a boundary component of $A_{i}$ and of $A_{j}$ for $1 \leqslant i<j \leqslant n$ is known to be either a Klein bottle or a torus. In Lemma 5.3 below we show that a map $f: K \rightarrow M$ constructed in a particular way is essential. 
LEMMA 5.3. Let $F$ be an incompressible surface properly embedded in a 3-manifold $M$ such that $\pi_{2}(M)=0$. Let $K$ be the union of a collection $A_{1}, \ldots$, $A_{n}$ of annuli such that each component of $A_{i} \cap A_{j}$ is a boundary component of $A_{i}$ and $A_{j}$ for $1 \leqslant i<j \leqslant n$. Suppose $f: K \rightarrow M$ is a map such that

(1) $f^{-1}(F)=\bigcup_{i=1}^{n} \partial A_{i}$.

(2) $f \mid A_{i}:\left(A_{i}, \partial A_{i}\right) \rightarrow(M, F)$ is not homotopic rel $\partial A_{i}$ to a map into $F$ for $i=1, \ldots, n$.

(3) There is an essential simple loop $\lambda_{1}$ in $K-\bigcup_{i=1}^{n} \partial A_{i}$ such that $f\left(\lambda_{1}\right)$ is essential in $M$.

Then $f_{*}: \pi_{1}(K) \rightarrow \pi_{1}(M)$ is monic.

Proof. Let $\lambda_{2}$ be a simple loop on $K$ that meets each component of $\bigcup_{i=1}^{n} \partial A_{i} \cup \lambda_{1}$ in a single point and crosses that component at the point of intersection. Now each element $\sigma \in \pi_{1}(K)$ is represented by a loop on $\lambda_{1} \cup \lambda_{2}$. Suppose $\sigma \in \pi_{1}(K)$ and $f_{*}(\sigma)=1$. Then there is a map $\phi: S^{1} \rightarrow K$ such that

(1) $\left[\phi\left(S^{1}\right)\right]=\sigma$.

(2) $\phi\left(S^{1}\right) \subset \lambda_{1} \cup \lambda_{2}$.

(3) $\phi\left(S^{1}\right)$ is not homotopic in $K$ to a map $\phi_{1}: S^{1} \rightarrow \lambda_{1} \cup \lambda_{2}$ such that $\phi_{1}^{-1}\left(\bigcup_{i=1}^{n} \partial A_{i}\right)$ contains fewer points than $\phi^{-1}\left(\bigcup_{i=1}^{n} \partial A_{i}\right)$.

If the cardinality of $\phi^{-1}\left(\bigcup_{i=1}^{n} \partial A_{i}\right)$ is zero, $\phi\left(S^{1}\right)$ is homotopic to a multiple of $\lambda_{1}$; and since $f\left(\lambda_{1}\right)$ is essential, $\left[\phi\left(S^{1}\right)\right]=1 \in \pi_{1}(K)$. Otherwise $f \phi: S^{1}$ $\rightarrow M$ is an essential map.

Let $D$ be the unit disk so $\partial D=S^{1}$ and $\Phi: D \rightarrow M$ a map such that $\Phi \mid \partial D=$ f申. After the usual argument we may suppose that $\Phi^{-1}(F)$ is the union of a collection of arcs properly embedded in $D$ since $F$ is incompressible. But we can find $\beta_{1} \subset \Phi^{-1}(F)$ such that $\beta_{1}$ cuts off a disk $D_{1}$ on $D$ so that $D_{1} \cap \Phi^{-1}(F)=$ $\beta_{1}$. Let $\beta_{2}$ be $\partial D_{1}-\beta_{1}$. Now $\phi\left(\beta_{2}\right)$ is homotopic to a spanning arc of $A_{j}$ where $j$ is some integer such that $1 \leqslant j \leqslant n$. It can be seen that $f \phi\left(\beta_{2}\right)$ is homotopic rel its endpoints to $\Phi\left(\beta_{1}\right)$ across $\Phi\left(D_{1}\right)$. It is a consequence of Proposition 3.3 that $f \mid A_{j}$ is homotopic rel $\partial A_{j}$ to a map into $F$. This contradicts our hypotheses on $f$ and we conclude $\sigma=1$ and $f_{*}$ is monic. This completes the proof of Lemma 5.3.

LemmA 5.4. Let $M$ be a 3-manifold such that $\pi_{2}(M)=0$ and $\partial M$ is incompressible and let $f: T \rightarrow M$ be a W-essential map. Then there is an essential embedding $g:(A, \partial A) \rightarrow(M, \partial M)$.

Proof. Since $f$ is $W$-essential there is an element $\sigma \in \pi_{1}(T)$ such that $f_{*}(\sigma)$ has a representative loop $l$ that is freely homotopic to a loop in $\partial M$. Let $\lambda_{1}$ and $\lambda_{2}$ be simple loops on $T$ that meet in a single point and cross at that point. Now $\sigma=k_{1}\left[\lambda_{1}\right]+k_{2}\left[\lambda_{2}\right]$ where $k_{1}$ and $k_{2}$ are integers. Let $(\widetilde{T}, q)$ be the finite 
sheeted covering space of $T$ associated with the subgroup of $\pi_{1}(T)$ generated by $\left[\lambda_{1}\right]$ and $k_{2}\left[\lambda_{2}\right]$. Then $(f q)_{*}: \pi_{1}(\widetilde{T}) \rightarrow \pi_{1}(M)$ is one-one and there is a simple loop $\mu$ on $\widetilde{T}$ such that $[q \mu]=\sigma$. Since $l$ is homotopic to a loop in $\partial M$, fq is homotopic to a map $f_{1}: \widetilde{T} \rightarrow M$ such that $f_{1}(\mu) \subset \partial M$. Now $f_{1}$ induces a map $\bar{f}_{1}:(A, \partial A) \rightarrow\left(M, f_{1}(\mu)\right)$ in a natural way. If $\bar{f}_{1}$ is not an essential map, $\overline{f_{1}}:(A, \partial A) \rightarrow(M, \partial M)$ is homotopic rel $\partial A$ to a map into $\partial M$ by Proposition 3.3. It follows that each representative of $f_{*}\left[\lambda_{1}\right]$ is freely homotopic to a loop in $\partial M$. Thus we may suppose that $f\left(\lambda_{1}\right) \subset \partial M$. But then $f$ induces a map $f_{2}:(A, \partial A) \rightarrow(M, \partial M)$ in a natural way and $f_{2}$ must be essential since $f$ was not homotopic to a map into $\partial M$. An application of Theorem 2 completes the proof of Lemma 5.4.

Let $F$ be a good surface in $M$. The next three lemmas will be used in the proof of the torus theorem in showing that if $f: T \rightarrow M$ is an essential map, we may suppose that $f$ is not homotopic to a map $f_{1}$ such that $f_{1}^{-1}(F)$ is empty.

LEMMA 5.5. Let $M_{1}$ be a submanifold of the 3-manifold $M$ and $\partial M_{1}$ incompressible in $M$. Let $g: T \rightarrow M_{1}$ be an essential embedding. Then $g(T)$ is essential in $M$.

Proof. Since $\partial M_{1}$ is incompressible, it is a consequence of standard geometric arguments that $\pi_{1}\left(M_{1}\right) \rightarrow \pi_{1}(M)$ is monic. Thus $g(T)$ is incompressible in $M$. Let $\lambda$ be a loop on $g(T)$. Suppose $f: A \rightarrow M$ is a free homotopy of $\lambda$ to a loop in $\partial M$. After the usual argument, we may suppose that $f^{-1}\left(\partial M_{1}\right)$ is the union of a collection of disjoint, simple essential loops. But then $\lambda$ is freely homotopic to a loop in $\partial M_{1}$. It follows that $g$ is an essential map. This completes the proof of Lemma 5.5.

LEMma 5.6. Let $M$ be an irreducible 3-manifold. Let $g:(A, \partial A) \rightarrow$ $(M, \partial M)$ be an essential embedding. Let $f: T \rightarrow M$ be an essential map such that there is no map $f_{1}$ homotopic to $f$ so that $f_{1}^{-1}(g(A))$ is empty. Then $M$ admits an embedding $h: T \rightarrow M$ such that $h_{*}$ is monic and $h$ is not homotopic to a map $h_{1}$ such that $h_{1}(T) \cap g(A)$ is empty. In fact $h^{-1} g(A)$ is the union of one, two, or four disjoint simple essential loops.

Proof. We may assume that $f^{-1}(g(A))$ is a collection of disjoint simple loops after a general position argument. We suppose that $f$ has been chosen so that $f^{-1}(g(A))$ contains as few loops as possible. Suppose some loop $\lambda$ in $f^{-1}(g(A))$ is inessential. Then $f(\lambda)$ is nullhomotopic on $g(A)$. Let $D$ be a regular neighborhood of the disk on $T$ bounded by $\lambda$. Then we can find a map $f_{1}: T \rightarrow$ $M$ such that $f_{1}|T-D=f| T-D$ and $f_{1}(D) \cap g(A)$ is empty. Since $\pi_{2}(M)=0$, $f$ and $f_{1}$ are homotopic. Thus the existence of $f_{1}$ contradicts our choice of $f$ and we suppose that each loop in $f^{-1} g(A)$ is essential. 
Let $A_{1}, \ldots, A_{n}$ be the closures of the components of $T-f^{-1} g(A)$. Let $R$ be a regular neighborhood of $g(A)$. Let $M_{1}$ be the manifold obtained by splitting $M$ along $g(A)$ and $P: M_{1} \rightarrow M$ the natural map.

Suppose that $f\left(A_{1}\right)$ meets both components of $R-g(A)$. We apply the theorem in [14] to find an embedding $h_{1}:(A, \partial A) \rightarrow\left(M_{1}, P^{-1} g(A)\right)$ such that $h_{1}(\partial A)$ meets both components of $P^{-1}(g(A))$ and $h_{1} *$ is monic. We may suppose that $P h_{1}\left(c_{1}\right)=P h_{1}\left(c_{2}\right)$. Then $P h_{1}$ defines an embedding $\bar{h}: K \rightarrow M$ where $K$ is a closed surface of genus 1 . Since $h_{1} \mid \alpha$ is not homotopic rel $\partial \alpha$ to a map into $P^{-1} g(A)$, it follows from the argument given in the proof of Lemma 5.3 that $\bar{h}_{*}: \pi_{1}(K) \rightarrow \pi_{1}(M)$ is monic. If $K$ is a torus we let $h=\bar{h}$. Otherwise the boundary of a regular neighborhood $N$ of $\bar{h}(K)$ is a torus embedded in $M$ since $M$ is orientable and $\partial N$ must be orientable. Since $\bar{h}_{*}$ is monic, $\pi_{1}(\partial N) \rightarrow \pi_{1}(M)$ is monic and any embedding $h: T \rightarrow \partial N$ has the desired properties. Thus we may suppose that $f\left(A_{i}\right)$ meets a single component of $R-g(A)$ for $i=1, \ldots, n$.

If $n=1, f$ is homotopic to a map $f_{1}$ such that $f_{1}(T) \cap g(A)$ is empty; so $n \geqslant 2$. We may suppose that $f\left(A_{1} \cup A_{2}\right)$ meets both components of $R-g(A)$. Let $M_{1}$ and $P$ be as above. Let $h_{j}:\left(A_{j}, \partial A_{j}\right) \rightarrow\left(M_{1}, P^{-1} g(A)\right)$ be the maps induced by $f$ for $j=1,2$. Then $h_{j}$ is not homotopic rel $\partial A_{j}$ to a map into $P^{-1} g(A)$ for $j=1,2$. Let $\alpha_{j}$ be a spanning arc of $A_{j}$ for $j=1,2$. It is a consequence of Theorem 2 that there exist embeddings $g_{j}:\left(A_{j}, \partial A_{j}\right) \rightarrow\left(M_{1}, P^{-1}(g(A))\right)$ that are $P^{-1} g(A)$-essential and $g_{j}\left(A_{j}\right)$ meets the same component of $P^{-1}(g(A))$ as does $h_{j}\left(A_{j}\right)$ for $j=1,2$. We suppose that $g_{1}$ and $g_{2}$ are in general position with respect to one another. Then $g_{1}\left(A_{1}\right) \cap g_{2}\left(A_{2}\right)$ is a collection of simple loops. We can remove any inessential loops by surgery. If any loops remain we can find an embedding $\bar{g}:(A, \partial A) \rightarrow\left(M_{1}, P^{-1} g(A)\right)$ such that $\bar{g}_{*}$ is monic and $\bar{g}(\partial A)$ meets both components of $P^{-1} g(A)$. In this case we obtain the desired result as above. Thus we may suppose that $g_{1}\left(A_{1}\right) \cap g_{2}\left(A_{2}\right)$ is empty and that $P g_{1}\left(\partial A_{1}\right)=P g_{2}\left(\partial A_{2}\right)$. Now $P g_{1}\left(A_{1}\right) \cup P g_{2}\left(A_{2}\right)$ induces an embedding $\bar{h}: K \rightarrow$ $M$ where $K$ is a surface of genus 1 . Since $g_{j}$ is $P^{-1} g(A)$-essential for $j=1,2$, $\bar{h}_{*}: \pi_{1}(K) \rightarrow \pi_{1}(M)$ is monic by Proposition 3.2 and Lemma 5.3. One now finds the embedding $h: T \rightarrow M$ as above. This completes the proof of Lemma 5.6.

Lemma 5.7. Let $f: T \rightarrow M_{1}$ be an essential map. Let $M_{j}, F_{j} \subseteq M_{j}$, $U\left(F_{j}\right) \subseteq M_{j}$ be a special hierarchy of $M_{1}$ with respect to $f$. Let $k$ be the smallest integer such that for every map $f_{1}$ homotopic to $f$, if $f_{1}(T) \subset M_{k}, f_{1}(T) \cap F_{k}$ is not empty. If $M_{k}$ admits an essential embedding $g$ of $A$ or $T, M_{1}$ admits an essential embedding of $A$ or $T$.

Proof. Suppose $g: T \rightarrow M_{k}$ is an essential embedding. Since $F_{k}$ is not a disk and $\partial M_{k}$ is incompressible in $M_{1}$, it is a consequence of 5.5 that $g(T)$ is 
essential in $M_{1}$. Thus we may suppose that $g:(A, \partial A) \rightarrow\left(M_{k}, \partial M_{k}\right)$ is an essential embedding. We may suppose $f(T) \subset M_{k}$. Since $g(A)$ could have been chosen as $F_{k}, g(A) \cap f(T)$ is not empty. We may suppose that $f^{-1}(g(A))$ is a collection of simple essential loops.

Suppose $\lambda$ is one of these loops and $f(\lambda)$ is freely homotopic to a loop in $\partial M_{1}$. Then $f: T \rightarrow M_{1}$ is $W$-essential and $M_{1}$ admits an essential embedding of an annulus by Lemma 5.4. Otherwise, we can find an embedding $h: T \rightarrow M_{k}$ such that $h_{*}$ is monic and $h(\lambda) \subset g(A)$ is freely homotopic to $g\left(c_{1}\right)$ in $g(A)$ by Lemma 5.6. Note that $h(\lambda)$ is not freely homotopic to a loop on $\partial M_{1}$ since $f(\lambda)$ is a multiple of $h(\lambda)$. It follows that $h: T \rightarrow M_{1}$ is essential. This completes the proof of Lemma 5.7.

LEMMA 5.8. Let $F$ be a surface properly embedded in $M$. Let $f:(A, \partial A)$ $\rightarrow(M, \partial M)$ be a map which is transverse with respect to $F$ such that $f^{-1}(F)$ is a collection of simple loops essential in $A$. Then we may assume that any embedding $g:(A, \partial A) \rightarrow(M, \partial M)$ constructed via a tower argument from $f$ meets $F$ in a collection of disjoint simple loops and that there is a fixed finite number of possibilities for $g(A) \cap F$ up to ambient isotopy in $F$. Furthermore the number of these possibilities is determined by the set $f^{-1}(F)$.

Proof. The proof of Lemma 5.8 is similar to that of Lemma 4.6 in [5] and we omit it.

LEMMA 5.9. Let $F$ be an incompressible surface in $\partial M$ and $F_{1}$ a planar submanifold of $F$ such that $\pi_{1}\left(F_{1}\right) \rightarrow \pi_{1}(F)$ is monic and $\chi\left(F_{1}\right)=-1$. Let $f:(A, \partial A) \rightarrow(M, F)$ be an F-essential map such that

(1) $f\left(c_{1}\right) \subset F_{1}$ and $f\left(c_{2}\right) \subset F-\partial F_{1}$.

(2) $f\left(c_{1}\right)$ is not freely homotopic on $F$ to a multiple of a loop in $\partial F_{1}$. Let $\lambda$ be a component of $\partial F_{1}$. Then there is an Fessential embedding $g:(A, \partial A) \rightarrow$ $(M, F)$ such that

(1) $g\left(c_{1}\right)=\lambda$.

(2) $g\left(c_{2}\right)$ lies in any prespecified neighborhood of $f\left(c_{2}\right)$.

Proof. Let $\left(M^{*}, p\right)$ be the covering space of $M$ associated with $\pi_{1}(F) \subset$ $\pi_{1}(M)$. Let $\bar{F}$ be a component of $p^{-1}(F)$ such that $p \mid \bar{F}$ is a homeomorphism. Let $\bar{f}_{1}:(A, \partial A) \rightarrow\left(M^{*}, \partial M^{*}\right)$ be a map such that $p \bar{f}_{1}=f$ and $\bar{f}_{1}\left(c_{1}\right) \subset \bar{F}_{1}=$ $p^{-1}\left(F_{1}\right) \cap \bar{F}$. Since $f(\alpha)$ is not homotopic rel its boundary to an arc on $F$ and $\pi_{1}(\bar{F}) \rightarrow \pi_{1}\left(M^{*}\right)$ is an isomorphism, $\bar{f}_{1}\left(c_{2}\right)$ does not lie on $\bar{F}$. It is a consequence of Theorem 3 that there is an embedding $\bar{g}_{1}:(A, \partial A) \rightarrow\left(M^{*}, \partial M^{*}\right)$ such that

(1) $\bar{g}_{1}\left(c_{1}\right)$ is freely homotopic in $\bar{F}$ to $\bar{F} \cap p^{-1}(\lambda)$.

(2) $\bar{g}_{1}\left(c_{2}\right)$ lies in any prespecified neighborhood of $\bar{f}_{1}\left(c_{2}\right)$.

After a homotopy, we may suppose that $\bar{g}_{1}\left(c_{1}\right)=\bar{F} \cap p^{-1}(\lambda)$. Let $g_{1}=p \bar{g}_{1}$. 
Then $g_{1}\left(c_{1}\right)=\lambda$ and $g_{1}\left(c_{2}\right)$ lies in a neighborhood of $f\left(c_{2}\right)$. Now the $\operatorname{arc} g_{1}(\alpha)$ is not homotopic to an arc on $F$ since the components of $\bar{g}_{1}(\partial \alpha)$ lie on distinct components of $p^{-1}(F)$. Lemma 5.9 can now be seen to be a consequence of Theorem 2.

LEMMA 5.10. Let $M$ be an irreducible 3-manifold with incompressible boundary. Let $F$ be an incompressible, boundary incompressible planar surface properly embedded in $M$ such that $\chi(F)=-1$. Let $f: T \rightarrow M$ be an essential map such that

(1) $f^{-1}(F)$ is a nonempty collection of disjoint simple essential loops.

(2) $f$ is not homotopic to a map $f_{1}: T \rightarrow M$ such that $f_{1}^{-1}(F)$ contains fewer components than $f^{-1}(F)$ and $f_{1}^{-1}(F)$ is the union of a collection of disjoint simple loops.

Then there is an essential embedding $g:(A, \partial A) \rightarrow(M, \partial M)$.

Proof. If $f$ is $W$-essential, Lemma 5.10 is a direct consequence of 5.4. Otherwise let $\lambda_{1}, \ldots, \lambda_{n}$ be the components of $f^{-1}(F)$. Since $f$ is $S$-essential, $f\left(\lambda_{i}\right)$ is not freely homotopic in $F$ to a loop in $\partial F$. We suppose that the closures of the components of $T-\bigcup_{i=1}^{n} \lambda_{i}$ are denoted by $A_{1}, \ldots, A_{n}$.

Let $R$ be a regular neighborhood of $F$. We divide the proof of Lemma 5.10 into two cases:

Case 1. $f\left(A_{1}\right)$ meets both components of $R-F$.

Case 2. $f\left(A_{1}\right)$ meets only one component of $R-F$. We now proceed with the proof of Case 1.

Let $M_{1}$ be the 3-manifold obtained by splitting $M$ along $F, \bar{f}: A \rightarrow M_{1}$ be the map induced by $f \mid A_{1}$ and $P: M_{1} \rightarrow M$ the natural projection map from $M_{1}$ onto $M$. Let $F_{j}$ be the component of $P^{-1}(F)$ such that $\bar{f}\left(c_{j}\right) \subset F_{j}$ for $j=1,2$. Note $F_{1} \neq F_{2}$. Let $\left(\widetilde{M}_{1}, p\right)$ be the covering space of $M_{1}$ associated with $\pi_{1}\left(F_{1}\right)$ $\subset \pi_{1}\left(M_{1}\right)$. We may suppose that $\widetilde{f}: A \rightarrow \widetilde{M}_{1}$ is a map such that $p \widetilde{f}=\bar{f}$. Let $\widetilde{F}_{j}$ be the component of $p^{-1}\left(F_{j}\right)$ on which $\widetilde{f}\left(c_{j}\right)$ lies for $j=1,2$. We may assume that $p \mid \widetilde{F}_{1}$ is a homeomorphism. Let $G$ be the component of $\partial \widetilde{M}_{1}$ which contains $\widetilde{F}_{1}$.

We claim that if every component of $\partial \widetilde{F}_{1}$ is freely homotopic in $G$ to a loop in $\widetilde{F}_{2}, \widetilde{M}_{1}$ is homeomorphic to $F \times[0,1]$.

Let $l_{1}, l_{2}$, and $l_{3}$ be the components of $\partial \widetilde{F}_{1}$. Suppose $h: l_{1} \times[0,1] \rightarrow$ $G$ is a free homotopy of $l_{1}$ to a loop in $\widetilde{F}_{2}$. We may suppose that $h^{-1} \partial\left(\widetilde{F}_{1} \cup \widetilde{F}_{2}\right)$ is a collection of disjoint simple loops $\mu_{1}, \ldots, \mu_{m}$ and $h\left(\mu_{1}\right)=l_{1}$. We may assume that $h$ has been chosen so that $m$ is minimal. Then $\mu_{i}$ is an essential loop in $l_{1} \times[0,1]$ as is $h\left(\mu_{i}\right)$ in $G$. We observe that $\partial \widetilde{F}_{2}$ is composed of disjoint simple loops and a number of copies of the real line. It can be seen that $h\left(\mu_{i}\right)$ must lie on one of the simple loops. Since any $\operatorname{arc}$ in $\widetilde{M}_{1}$ with its boundary on 
$\widetilde{F}_{1}$ is homotopic rel its boundary to an arc on $\widetilde{F}_{1}$ and since $\pi_{2}\left(\widetilde{M}_{1}\right)=0$, one can deform any free homotopy of pairs of loops in $\widetilde{F}_{1}$ until the free homotopy lies in $\widetilde{F}_{1}$. Since no pair of boundary components of $\widetilde{F}_{1}$ are freely homotopic in $\widetilde{F}_{1}$, no pair of boundary components of $\widetilde{F}_{1}$ are freely homotopic in $G$. Thus it can be seen that we may suppose that $h\left(\mu_{i}\right)$ does not meet $\widetilde{F}_{1}$ for $i=1, \ldots, m$ and $l_{1}$ is freely homotopic to a power of a boundary component $h\left(\mu_{2}\right)$ of $\widetilde{F}_{2}$. Note $h\left(\mu_{2}\right)$ is an essential loop in $\widetilde{F}_{2}$ and $l_{1} \cup h\left(\mu_{2}\right)$ bounds an annulus embedded in $G$.

Suppose that $l_{1}, l_{2}$, and $l_{3}$ are freely homotopic in $G$ to loops in $\widetilde{F}_{2}$. Then we can find disjoint simple loops $\mu_{1}, \mu_{2}$, and $\mu_{3}$ in $\partial \widetilde{F}_{2}$ such that $\mu_{j} \cup l_{j}$ bounds an annulus $\bar{A}_{j}$ embedded in $G$ for $j=1,2,3$. Since $\widetilde{F}_{2}$ is connected we can find disjoint simple arcs $\alpha_{1}$ and $\alpha_{2}$ properly embedded in $\widetilde{F}_{2} \cup \bar{A}_{1} \cup \bar{A}_{2} \cup \bar{A}_{3}$ such that

(1) $\alpha_{j} \cap \widetilde{F}_{2}$ is an arc properly embedded in $\widetilde{F}_{2}$ for $j=1,2$.

(2) $\alpha_{1}$ meets both $l_{1}$ and $l_{2}$.

(3) $\alpha_{2}$ meets both $l_{1}$ and $l_{3}$.

Note that $\alpha_{1}$ meets $\mu_{1}$ and $\mu_{2}$ and $\alpha_{2}$ meets $\mu_{1}$ and $\mu_{3}$. We observe that $\alpha_{1}$ and $\alpha_{2}$ are homotopic rel their boundaries to $\operatorname{arcs}$ on $\widetilde{F}_{1}$ since the natural map $\pi_{1}\left(\widetilde{F}_{1}\right)$ $\rightarrow \pi_{1}\left(\widetilde{M}_{1}\right)$ induced by inclusion is onto. It is a consequence of the proof of the loop theorem [12] that there are disjoint disks $D_{1}$ and $D_{2}$ properly embedded in $\widetilde{M}_{1}$ such that $D_{j} \cap \widetilde{F}_{1}$ is a simple arc properly embedded in $\widetilde{F}_{1}$ and $D_{j} \cap(G-$ $\left.\widetilde{F}_{1}\right)=\operatorname{int}\left(\alpha_{j}\right)$ for $j=1,2$. We observe that $\widetilde{F}_{1}-\left(D_{1} \cup D_{2}\right)$ is simply connected. Let $l \subset \operatorname{int}\left(\widetilde{F}_{2}\right)$ be a boundary component of a regular neighborhood of $\mu_{1} \cup$ $\mu_{2} \cup \mu_{3} \cup\left(\left(\alpha_{1} \cup \alpha_{2}\right) \cap \widetilde{F}_{2}\right)$. Now $l$ lies in the complement of $D_{1} \cup D_{2}$ and there is a free homotopy of $l$ to a loop in $\widetilde{F}_{1}$. It can be shown that this free homotopy can be assumed to lie in the complement of $D_{1} \cup D_{2}$ using an argument similar to one given above. It follows that $l$ is inessential in $\widetilde{M}_{1}$. Since $F$ is incompressible in $M, \widetilde{F}_{2}$ is incompressible in $\widetilde{M}_{1}$. Thus $l$ is null-homotopic on $\widetilde{F}_{2}$ and $l$ bounds a disk on $\widetilde{F}_{2}$. It follows that $\widetilde{F}_{2}$ is homeomorphic to $F$. Let $B$ be the manifold obtained by removing the interior of a regular neighborhood of $D_{1} \cup D_{2}$ from $\widetilde{M}_{1}$. Then one component of $\partial B$ is a 2-sphere. Since $\pi_{2}\left(\widetilde{M}_{1}\right)=0$, $B$ is a homotopy 3 -cell. It can now be seen that $p: \widetilde{M}_{1} \rightarrow M_{1}$ is a homeomorphism and that $\widetilde{M}_{1}$ is homeomorphic to $F \times I$. This establishes our claim.

We suppose that $\widetilde{M}_{1}$ is not homeomorphic to $F \times[0,1]$. As a consequence of our claim above, it follows that we may assume that the loop $l_{1}$ in $\partial \widetilde{F}_{1}$ is not freely homotopic in $G$ to a loop in $\widetilde{F}_{2}$. It is a consequence of Theorem 3 that the existence of $\widetilde{f}: A \rightarrow \widetilde{M}_{1}$ guarantees the existence of an embedding $\widetilde{g}_{1}: A \rightarrow$ $\widetilde{M}_{1}$ such that

(1) $\widetilde{g}_{1}\left(c_{j}\right) \subset \widetilde{F}_{j}$ for $j=1,2$.

(2) $\widetilde{g}_{1}\left(c_{1}\right)$ is freely homotopic to $l_{1}$. 
(3) $\tilde{g}_{1 *}: \pi_{1}(A) \rightarrow \pi_{1}\left(\widetilde{F}_{j}\right)$ is monic.

Since $\widetilde{g}_{1}\left(c_{1}\right)$ is simple, we may assume that $\tilde{g}_{1}\left(c_{1}\right)=l_{1}$. Since $p \mid \widetilde{F}_{1}$ is a homeomorphism, $p \tilde{g}_{1} \mid c_{1}$ is a homeomorphism.

If $p \widetilde{g}_{1}\left(c_{2}\right)$ is freely homotopic to a loop in $\partial F_{2}$, we assume that $p \tilde{g}_{1}\left(c_{2}\right) \subset$ $\partial F_{2}$ and will show below that $P p \tilde{g}_{1}:(A, \partial A) \rightarrow(M, \partial M)$ is an essential map. In this case Lemma 5.10 is a consequence of Theorem 2.

If $\tilde{g}_{1}\left(c_{2}\right)$ is not freely homotopic to a loop in $\partial F_{2}$, we replace $p \tilde{g}_{1}$ by another map and proceed as has been indicated above.

Suppose $p \tilde{g}_{1}\left(c_{2}\right)$ is not freely homotopic in $F_{2}$ to a loop in $\partial F_{2}$. Let $\left(\bar{M}_{1}, q\right)$ be the covering space of $M_{1}$ associated with $\pi_{1}\left(F_{2}\right) \subset \pi_{1}\left(M_{1}\right)$. Let $\bar{g}_{1}$ : $A \rightarrow \bar{M}_{1}$ be a map such that $q \bar{g}_{1}=p \tilde{g}_{1}$. Let $\bar{F}_{j}$ be the component of $q^{-1}\left(F_{j}\right)$ such that $\bar{g}_{1}\left(c_{j}\right) \subset \bar{F}_{j}$ for $j=1,2$. Let $\bar{G}$ be the component of $\partial \bar{M}_{1}$ which contains $\bar{F}_{2}$. We may suppose $q \mid \bar{F}_{2}$ is a homeomorphism. The proof of Lemma 5.10 is the same in case $\bar{M}_{1}$ is homeomorphic to $\bar{F}_{2} \times[0,1]$ as it is in case $\widetilde{M}_{1}$ is homeomorphic to $\widetilde{F}_{1} \times[0,1]$. It follows from the claim above that we may assume some component $\mu_{1}$ of $\partial \bar{F}_{2}$ is not homotopic in $\bar{G}$ to a loop in $\partial \bar{F}_{1}$. As above by Theorem 3 , we can find an embedding $\bar{g}_{2}: A \rightarrow \bar{M}_{1}$ such that

(1) $\bar{g}_{2}\left(c_{2}\right)=\mu_{1}$.

(2) $\bar{g}_{2}\left(c_{1}\right)$ lies in a small neighborhood in $\partial \bar{M}_{1}$ of $\bar{g}_{1}\left(c_{1}\right)$.

(3) $\bar{g}_{2 *}: \pi_{1}(A) \rightarrow \pi_{1}\left(\bar{M}_{1}\right)$ is monic.

Since $\bar{g}_{1}\left(c_{1}\right)$ is simple, its neighborhood may be taken to be an annulus and we assume $\bar{g}_{2}\left(c_{1}\right)=\bar{g}_{1}\left(c_{1}\right)$. Now $q \mid \bar{F}_{2}$ and $q \mid \bar{g}_{1}\left(c_{1}\right)$ are homeomorphisms so $q \bar{g}_{2} \mid \partial A$ is an embedding and thus $P q \bar{g}_{2}(\partial A) \subset \partial F$. Clearly we may as well suppose $P p \tilde{g}_{1}(\partial A) \subset \partial F$. Let $h=P p \tilde{g}_{1}:(A, \partial A) \rightarrow(M, \partial M)$. We claim $h$ is an essential map.

If not, the arc $h(\alpha)$ is homotopic rel its boundary to an arc in $\partial M$. Since $\partial M$ is incompressible and $\pi_{2}(M)=0, h$ is homotopic rel $\partial A$ to a map $h_{1}: A \rightarrow$ $\partial M$ by Proposition 3.2. Let $H: A \times[0,1] \rightarrow M$ be a homotopy such that

(1) $H(x, 0)=h(x)$ for $x \in A$.

(2) $H(x, 1)=h_{1}(x)$ for $x \in A$.

(3) $H(x, t)=h(x)$ for $x \in \partial A$ and $t \in[0,1]$.

After a general position argument, we may suppose that $H^{-1}(F)$ is an incompressible surface properly embedded in $A \times[0,1]$. We suppose that $h_{1}$ has been chosen so that the number of components in $h_{1}^{-1}(F)$ is minimal. We observe that $H^{-1}(F)$ must be a disjoint union of disks and annuli. Suppose $D$ is a disk in $H^{-1}(F)$. Since $h^{-1}(F)=\partial A, D \cap \partial(A \times[0,1]) \subset A \times\{1\}$. Of course $H(\partial D)$ lies on one component of $\partial F$. Since $F$ is incompressible, it can be seen that $h_{1}$ was not chosen so that the number of components in $h_{1}^{-1}(\partial F)$ would be minimal. Suppose $A_{1}$ is an annulus in $H^{-1}(F)-\partial A \times[0,1]$. Then both components of 
$H\left(\partial A_{1}\right)$ lie on $\partial F$. Since $H\left(A_{1}\right) \subset F$ and each component of $\partial A_{1}$ is an essential loop in $A \times[0,1], H \mid A_{1}$ is homotopic rel $\partial A_{1}$ to a map into $\partial F$. Thus we may suppose that $H\left(A_{1}\right) \subset \partial F$. Note that $A_{1}$ divides $A \times[0,1]$ into two solid tori, and that the restriction of $H$ to the one of them which meets $A \times\{0\}$ defines a homotopy of $h$ to a map $h_{2}$. It can be seen that the number of components in $h_{2}^{-1}(F)$ is one less than that of $h_{1}^{-1}(F)$. It follows that the best possible choice for $h_{1}$ was not made. We conclude that $H^{-1}(F)=\partial A \times[0,1]$.

We may now consider $H$ as a map into $M_{1}$. Since $H$ can be lifted to a map $\widetilde{H}: A \times[0,1] \rightarrow \widetilde{M}_{1}$, we see that $\tilde{g}_{1}\left(c_{1}\right)$ is homotopic to $\tilde{g}_{1}\left(c_{2}\right)$ in $\partial \widetilde{M}_{1}$. This contradicts our hypothesis that $l_{1}$ was not freely homotopic in $G$ to a loop in $\widetilde{F}_{2}$ and our claim is established.

In this case Lemma 5.10 follows from Theorem 2 above.

Suppose that $\widetilde{M}_{1}$ is homeomorphic to $F \times[0,1]$. Since the natural map from $\pi_{1}\left(\widetilde{F}_{1}\right)$ into $\pi_{1}\left(\widetilde{M}_{1}\right)$ induced by inclusion is an isomorphism and $p: \widetilde{M}_{1} \rightarrow$ $M_{1}$ is a homeomorphism, it is a consequence of Theorem 3.1 in [1] that there is a homeomorphism $\theta: F \times[0,1] \rightarrow M_{1}$ such that

(1) $\theta(F \times[0,1])=M_{1}$.

(2) $\theta(F \times\{0\})=F_{1}$.

(3) $\theta(F \times\{1\})=F_{2}$.

Now $h_{j}=P \mid F_{j}$ is a homeomorphism for $j=1,2$. Let $\rho_{1}: F \times\{0\} \rightarrow F \times\{1\}$ be the homeomorphism defined by $\rho_{1}=\theta^{-1} h_{2}^{-1} h_{1} \theta$. Let $\rho: F \rightarrow F$ be the homeomorphism defined by $\rho(x)=y$ if $\rho_{1}(x, 0)=(y, 1)$. Let $\beta_{0}$ be a simple separating arc properly embedded in $F$. We require that neither component of the complement of $\beta_{0}$ is simply connected. Let $\beta_{n}=\rho^{n} \beta_{0}$ for $n$ a positive integer. Then it can be seen that there exists an integer $k$ such that $\beta_{0}$ and $\beta_{k}$ are homotopic under a map $H:[0,1] \times[0,1]$ such that

(1) $H([0,1] \times\{0\})=\beta_{0}$.

(2) $H([0,1] \times\{1\})=\beta_{k}$.

(3) $H(\{0,1\} \times[0,1]) \subset \partial F$.

One then defines an essential map $g_{0}:(A, \partial A) \rightarrow(M, \partial M)$ as follows: Let $D_{i}=\beta_{i} \times[0,1]$ for $i=0, \ldots, k-1$. Let $D_{k}=[0,1] \times[0,1]$. Note that $P \theta\left(\bigcup_{i=0}^{k-1} D_{i}\right)$ together with $H\left(D_{k}\right) \subset F$ defines a map $g_{0}:(A, \partial A) \rightarrow(M, \partial M)$ in a natural way. Furthermore this map is essential since it is a consequence of Lemma 3.1 that $P \theta\left(\beta_{0} \times\{0\}\right)$ is not homotopic rel its boundary to an arc in $\partial M$ for $F$ is boundary incompressible in $M$.

We may now suppose that $f\left(A_{1}\right)$ meets only one component of $R-F$. We again let $M_{1}$ be the manifold obtained by splitting $M$ along $F$. We define $P, F_{1}$, and $F_{2}$ as above. Now $f \mid A_{1}$ induces a map $\bar{f}: A \rightarrow M_{1}$ and we may suppose that $\bar{f}(\partial A) \subset F_{1}$. We observe that it is a consequence of Proposition 3.2 that 
$\bar{f}(\alpha)$ is not homotopic rel $\bar{f}(\partial \alpha)$ to an arc in $F_{1}$ since $\pi_{2}(M)=0$ and $f \mid A_{1}$ is not homotopic rel $\partial A_{1}$ to a map into $F$. Let $\left(\widetilde{M}_{1}, p\right)$ be the covering space of $M_{1}$ associated with $\pi_{1}\left(F_{1}\right) \subset \pi_{1}\left(M_{1}\right)$. Let $\widetilde{F}_{1}$ be a component of $p^{-1}\left(F_{1}\right)$ such that $p \mid \widetilde{F}_{1}$ is a homeomorphism. Then there is a map $\widetilde{f}: A \rightarrow \widetilde{M}_{1}$ such that $p \widetilde{f}=\bar{f}$ and $\widetilde{f}\left(c_{1}\right) \subset \widetilde{F}_{1}$. Let $\widetilde{F}_{2}$ be the component of $p^{-1}\left(F_{1}\right)$ on which $\widetilde{f}\left(c_{2}\right)$ lies.

Note that if $\widetilde{F}_{1}=\widetilde{F}_{2}, \widetilde{f}(\alpha)$ is homotopic rel its boundary to an arc in $\widetilde{F}_{1}$ and by Proposition $3.2, \widetilde{f}$ is homotopic rel $\partial A$ to a map into $\widetilde{F}_{1}$. This would contradict condition (2) in the statement of the lemma.

As above we may suppose that some component $l_{1}$ of $\partial \widetilde{F}_{1}$ is not freely homotopic in $\partial \widetilde{M}_{1}$ to a loop in $\widetilde{F}_{2}$ for otherwise $\widetilde{M}_{1}$ is homeomorphic to $\widetilde{F}_{1} \times$ $[0,1]$. We can apply Theorem 3 to find an embedding $\widetilde{f}_{1}: A \rightarrow \widetilde{M}_{1}$ such that $\widetilde{f}_{1}\left(c_{1}\right)=l_{1}$ and $\widetilde{f}_{1}\left(c_{2}\right) \subset \widetilde{F}_{2}$. Thus $p \widetilde{f}_{1}\left(c_{1}\right) \subset \partial F_{1}$. If $p \widetilde{f}_{1} \mid c_{2}: c_{2} \rightarrow$ $F_{1}$ is not an embedding, we reverse the roles of $c_{1}$ and $c_{2}$ as above to obtain an embedding ${\widetilde{f_{2}}}_{2}: A \rightarrow \widetilde{M}_{1}$ such that

(1) $p \widetilde{f}_{2} \mid c_{j}$ is an embedding for $j=1,2$.

(2) $p \widetilde{f}_{2}(\partial A) \subset \partial F_{1}$.

(3) $\widetilde{f}_{2}\left(c_{2}\right) \subset \widetilde{F}_{1}$.

(4) $\widetilde{f}_{2}\left(c_{1}\right)$ does not lie on $\widetilde{F}_{1}$.

(5) $\widetilde{f}_{2}\left(c_{1}\right)$ is not freely homotopic to $\widetilde{f}_{2}\left(c_{2}\right)$ in $\partial \widetilde{M}_{1}$.

Let $h=P p \widetilde{f}_{2}$. We claim that $h$ is an essential map. Suppose that $h(\alpha)$ is homotopic rel its boundary to an arc in $\partial M$. Since $M$ has incompressible boundary and $\pi_{2}(M)=0$, there is a map $H: A \times[0,1] \rightarrow M$ such that

(1) $H(x, 0)=h(x)$ for $x \in A$.

(2) $H(A \times\{1\}) \subset \partial M$.

(3) $H(x, t)=h(x)$ for $x \in \partial A$ and $t \in[0,1]$.

We suppose that $H$ has been chosen so that $H^{-1}(F)$ is a system of incompressible surfaces in $A \times[0,1]$ and $H^{-1}(F)$ contains as few components as possible. One sees as above that $H^{-1}(F)=\partial A \times[0,1]$. But then $\widetilde{f}_{2}$ is homotopic rel $\partial A$ to a map into $\partial \widetilde{M}_{1}$. This contradicts our construction of $\widetilde{f}_{2}$. Thus $h$ is essential and the proof of Lemma 5.10 is completed by an application of Theorem 2 above.

LEMMA 5.11. Let $F$ be a good surface and $f: T \rightarrow M$ an essential map. Let $N$ be a regular neighborhood of $F$. Suppose

(1) $f^{-1}(F)$ is the union of a nonempty collection of simple loops.

(2) $f$ is not homotopic to a map $\bar{f}: T \rightarrow M$ such that $\bar{f}^{-1}(F)$ is the union of a collection of simple loops and $\bar{f}^{-1}(F)$ contains fewer components that $f^{-1}(F)$.

(3) The $f$ image of each component of $T-f^{-1}(F)$ meets both components of $N-F$.

Then there is either an essential map $f_{1}: K \rightarrow M$ where $K$ is either a torus or Klein bottle such that the restriction of $f_{1}$ to each component of $f_{1}^{-1}(F)$ and 
each component of $K-f_{1}^{-1}(F)$ is a homeomorphism or an essential embedding $g:(A, \partial A) \rightarrow(M, \partial M)$. If the former is the case, $f_{1}^{-1}(F)$ is not empty.

Proof. As a consequence of Lemma 5.10, we may assume that $F$ is not the planar surface with three boundary components. It follows from Lemma 5.4 that we may assume that for each loop $\lambda$ in $f^{-1}(F), f(\lambda)$ is not freely homotopic to a loop in $\partial F$.

Let $M \mid F$ be the 3-manifold obtained by splitting $M$ along $F$. Let $P$ : $M \mid F$ $\rightarrow M$ be the natural projection map. Let $F^{1}$ and $F^{2}$ be the components of $P^{-1}(F)$. Let $M_{i}$ be homeomorphic to $M \mid F$ and $F_{i}^{1}$ and $F_{i}^{2}$ be the copies of $F^{1}$ and $F^{2}$ respectively in $\partial M_{i}$ for $i$ an integer. Let $(\widetilde{M}, p)$ be the infinite cyclic covering space of $M$ obtained from $\bigcup_{i=-\infty}^{\infty} M_{i}$ by identifying $F_{i}^{1}$ and $F_{i+1}^{2}$ for $i$ an integer. We denote the image of $F_{i}^{1}$ under this identification by $F_{i}$ are are careful to define the identification so that the natural projection map from $\widetilde{M}-$ $\bigcup_{i=-\infty}^{\infty} F_{i}$ to $M$ can be extended to the covering map $p: \widetilde{M} \rightarrow M$. Let $(\widetilde{T}, q)$ be the infinite cyclic covering of $T$ associated with some simple essential loop in $f^{-1}(F)$. Then there is a map $\widetilde{f}: \widetilde{T} \rightarrow \widetilde{M}$ such that $p \widetilde{f}=f q$.

Let $N_{i}$ be the submanifold of $\widetilde{M}$ bounded by $F_{0}$ and $F_{i}$ for each positive integer $i$. Then $\widetilde{f}^{-1}\left(N_{i}\right)$ is an annulus $A_{i}$ such that $\widetilde{f}\left(\partial A_{i}\right)$ meets both $F_{0}$ and $F_{i}$. It is a consequence of Lemma 5.8 that an annulus embedded in $N_{i}$ constructed via a tower argument from the map $\widetilde{f} \mid A_{i}$ can meet $F_{j}$ in only finitely many ways up to free homotopy since $\widetilde{f}\left(A_{i}\right) \cap F_{j} \subset p^{-1}(f(T) \cap F)$ where $0 \leqslant j \leqslant i$. Of course the number $n_{0}$ determined by $f(T) \cap F$ is independent of both $i$ and $j$ above. Let $n=2 n_{0}+2$. Let $f_{n}=\widetilde{f} \mid A_{n}: A_{n} \rightarrow N_{n}$.

It is a consequence of Theorem 3 that we can find an embedding $g_{n}$ : $\left(A_{n}, \partial A_{n}\right) \rightarrow\left(N_{n}, \partial N_{n}\right)$ such that one component of $g_{n}\left(\partial A_{n}\right)$ is not freely homotopic in $F_{0} \cup F_{n}$ to a loop in $\partial\left(F_{0} \cup F_{n}\right)$ since $F$ is not the planar surface with three boundary components. Suppose a loop $\lambda$ in $g_{n}^{-1}\left(\bigcup_{i=1}^{n-1} F_{i}\right)$ is inessential on $A_{n}$. Then $g_{n}(\lambda)$ bounds a disk in $\bigcup_{i=1}^{n-1} F_{i}$ and it follows from standard arguments that $g_{n}$ can be modified to obtain a map $g_{n}^{1}:\left(A_{n}, \partial A_{n}\right) \rightarrow\left(N_{n}, \partial N_{n}\right)$ which agrees with $g_{n}$ on all essential loops in $g_{n}^{-1}\left(\bigcup_{i=1}^{n-1} F_{i}\right)$ so that $g_{n}^{1}\left(A_{n}\right) \cap$ $\bigcup_{i=0}^{n} F_{i}$ contains fewer loops than does $g_{n}\left(A_{n}\right) \cap \bigcup_{i=0}^{n} F_{i}$. It follows that we may suppose that all loops in $g_{n}^{-1}\left(\bigcup_{i=0}^{n} F_{i}\right)$ are essential in $A_{n}$. After a general position argument we may suppose that $g_{n}\left(A_{n}\right)$ crosses $F_{i}$ at each loop in $g_{n}\left(A_{n}\right)$ $\cap F_{i}$.

Let $\lambda$ be a loop in $g_{n}^{-1}\left(F_{i}\right)$ for $0<i<n$. Then by Lemma $5.1, p g_{n}(\lambda)$ is not freely homotopic in $F$ to a loop in $\partial F$ since $F$ is good and $p g_{n}(A)$ shows that $p g_{n}(\lambda)$ is freely homotopic in $M$ to a loop in $F$ which is not freely homotopic in $F$ to a loop in $\partial F$.

We observe that $n$ has been chosen so large that there are integers $k, l, m$ such that 
(1) $0<k<l<m<n$.

(2) $p\left(g_{n}\left(A_{n}\right) \cap F_{k}\right)=p\left(g_{n}\left(A_{n}\right) \cap F_{l}\right)=p\left(g_{n}\left(A_{n}\right) \cap F_{m}\right)$.

Thus there are distinct loops $\lambda_{1}, \lambda_{2}$, and $\lambda_{3}$ on $A_{n}$ such that $p g_{n}\left(\lambda_{1}\right)=p g_{n}\left(\lambda_{2}\right)$ $=p g_{n}\left(\lambda_{3}\right)$ and $g_{n}\left(\lambda_{j}\right) \subset F_{k} \cup F_{1} \cup F_{m}$ for $j=1,2,3$. We may suppose that $\lambda_{3}$ lies between $\lambda_{1}$ and $\lambda_{2}$ on $A_{n}$. Let $B_{j}$ be the annulus on $A_{n}$ bounded by $\lambda_{j} \cup \lambda_{3}$ for $j=1,2$ and $B_{3}=B_{1} \cup B_{2}$. Let $R$ be a regular neighborhood of $F$ in $M$. It follows from a simple argument that there exists a $j=1,2$, or 3 such that the image under $p g_{n}$ of every neighborhood in $B_{j}$ of $\partial B_{j}$ meets both components of $R-F$. We suppose that this is the case for $B_{1}$.

Let $\mu_{1}, \ldots, \mu_{m}$ be the components of $g_{n}^{-1}\left(\bigcup_{i=1}^{n-1} F_{i}\right) \cap B_{1}$. Let $C_{1}, \ldots$, $C_{m-1}$ be the closures of the components of $B_{1}-\bigcup_{i=1}^{m} \mu_{i}$. If $g_{n}\left(\partial C_{i}\right)$ lies on a single component $F_{j}$ of $p^{-1}(F)$ and $g_{n} \mid C_{i}:\left(C_{i}, \partial C_{i}\right) \rightarrow\left(\widetilde{M}, F_{j}\right)$ is homotopic rel $\partial C_{i}$ to a map into $F_{j}$, we can replace $g_{n}$ by an embedding $g_{n}^{1}: B_{1} \rightarrow \widetilde{M}$ such that $g_{n}\left|\partial B_{1}=g_{n}^{1}\right| \partial B_{1}$ and $g_{n}^{1}\left(B_{1}\right) \cap \bigcup_{i=0}^{n} F_{i}$ contains fewer loops than does $g_{n}\left(B_{1}\right) \cap \bigcup_{i=0}^{n} F_{i}$. Thus we may suppose that $g_{n} \mid C_{i}:\left(C_{i}, \partial C_{i}\right) \rightarrow\left(\tilde{M}, F_{j}\right)$ is not homotopic rel $\partial C_{i}$ to a map into $F_{j}$ since the components of $g_{n}\left(\partial B_{1}\right)$ lie on distinct components of $p^{-1}(F)$.

Let $\bar{f}: K \rightarrow M$ be the map induced by $p g_{n}: B_{1} \rightarrow M$. By construction the restriction of $\bar{f}$ to each component of $K-\bar{f}^{-1}(F)$ is an embedding. We will show that $\bar{f}$ is essential.

Let $\lambda$ be any simple loop in $\bar{f}^{-1}(F)$. Then $f(\lambda)$ is an essential simple loop on $F$ that is not freely homotopic to a loop in $\partial F$ by construction. Since $F$ is good, $f(\lambda)$ is not freely homotopic to a loop in $\partial M$. It follows from Lemma 5.3 and the construction of $\bar{f}$ that $\bar{f}_{*}: \pi_{1}(K) \rightarrow \pi_{1}(M)$ is monic. Thus $\bar{f}$ is essential and the proof of Lemma 5.11 is complete.

LEMMA 5.12. Let $M$ be a connected, irreducible 3-manifold such that $\partial M$ is incompressible. Let $K_{1}$ be a closed connected surface such that $\chi\left(K_{1}\right)=0$. Let $F_{1}$ be a good surface in $M$ that is not the planar surface with three boundary components. Let $f: K_{1} \rightarrow M$ be a map such that

(1) $f_{*}: \pi_{1}\left(K_{1}\right) \rightarrow \pi_{1}(M)$ is monic.

(2) $f^{-1}\left(F_{1}\right)$ is the union of a collection of disjoint simple loops.

(3) There is a loop $\lambda \subset f^{-1}\left(F_{1}\right)$ such that $f(\lambda)$ is not freely homotopic in $F_{1}$ to a loop in $\partial F_{1}$.

(4) The components of $K_{1}-f^{-1}\left(F_{1}\right)$ are open annuli whose closures we denote by $A_{1}, \ldots, A_{n}$.

(5) $f\left(A_{1}\right)$ meets only one component of $R-F_{1}$ (where $R$ is a regular neighborhood of $F$ in $M$ ).

(6) $f \mid A_{i}$ is not homotopic rel $\partial A_{i}$ to a map into $F_{1}$ for $i=1, \ldots, n$.

(7) $f$ is not homotopic to a map $f_{1}$ such that $f_{1}^{-1}\left(F_{1}\right)$ contains fewer loops than does $f^{-1}\left(F_{1}\right)$. 
Then there is a closed connected surface $K$ such that $\chi(K)=0$ and an essential embedding $\bar{f}: K \rightarrow M$ such that $\bar{f}^{-1}\left(F_{1}\right)$ contains an essential simple loop $\lambda$ so that $\bar{f}(\lambda)$ is not freely homotopic in $F_{1}$ to a loop in $\partial F_{1}$.

Proof. Let $g_{i}:(A, \partial A) \rightarrow\left(M, F_{1}\right)$ for $i=1, \ldots, m$ be a maximal collection of pairwise disjoint $F_{1}$-essential embeddings such that

(1) $\bigcup_{i=1}^{m} g_{i}(A)$ meets only one component of $R-F_{1}$.

(2) Neither $g_{i}\left(c_{1}\right)$ nor $g_{i}\left(c_{2}\right)$ is freely homotopic in $F_{1}$ to a loop in $\partial M_{1}$ for $i=1, \ldots, m$.

(3) $g_{i}(A)$ and $g_{j}(A)$ are not parallel rel $F_{1}$ for $1 \leqslant i<j \leqslant m$.

The existence of this collection, in particular its finiteness is guaranteed by the theorem on p. 60 in [15]. Let $R_{1}$ be a regular neighborhood of $\bigcup_{i=1}^{m} g_{i}(A) \cup$ $F_{1}$. Let $M_{1}$ and $M_{2}$ be the closures of the components of $R_{1}-F_{1}$. We may suppose that $M_{2}$ is homeomorphic to $F_{1} \times[0,1]$. Since $F_{1}$ does not separate $M$ and $\left[\operatorname{cl}\left(\partial M_{1}-F_{1}\right)\right]$ is homologous to $\left[F_{1}\right]$, there is a component of $\partial M_{1} \cap$ $\operatorname{int}(M)$ whose closure fails to separate $M$. We denote the closure of this component by $F_{2}$. It can be seen that $\chi\left(F_{2}\right) \geqslant \chi\left(F_{1}\right)$. It is a consequence of repeated applications of Lemma 5.2 or of the proof of Lemma 5.2 that $F_{2}$ is good. Thus $\chi\left(F_{2}\right)=\chi\left(F_{1}\right)$. Now if $T_{0}$ is the closure of a component of $\partial M_{1}-\left(\partial M \cup F_{1} \cup\right.$ $\left.F_{2}\right)$ it can be seen that $\chi\left(T_{0}\right)=0$ since $\chi\left(\partial M_{1}\right)=2 * \chi\left(F_{1}\right)$ and $T_{0} \neq S^{2}$.

Let $R_{2}$ be a regular neighborhood of $\bigcup_{i=1}^{m} g_{i}(A)$ in $M_{1}$. Let $\alpha_{i}$ be a spanning arc of $A_{i}$ for $i=1, \ldots, n$. Since $f \mid A_{i}$ is not homotopic rel $\partial A_{i}$ to a map into $F_{1}$ for $i=1, \ldots, n, f\left(\alpha_{i}\right)$ is not homotopic rel its endpoints to an arc in $F_{1}$ for $i=1, \ldots, n$ by Proposition 3.2.

Suppose that $\bigcup_{i=1}^{m} g_{i}(A) \cup f\left(A_{j}\right)$ meets only one component of $N-F_{1}$. We claim that $f$ is homotopic to a map $f_{1}$, under a homotopy constant outside of a neighborhood of $A_{j}$, such that

(1) $f_{1}^{-1}\left(F_{1}\right)=f^{-1}\left(F_{1}\right)$.

(2) $f_{1}^{-1}\left(\bigcup_{i=1}^{m} g_{i}(\partial A)\right) \cap A_{j}$ is empty.

We need only show that there is a homotopy $h_{t}:\left(A_{j}, \partial A_{j}\right) \rightarrow\left(M, F_{1}\right)$ for $t \in$ $[0,1]$ such that

(1) $h_{0}=f \mid A_{j}$.

(2) $h_{1}\left(\partial A_{j}\right) \cap \bigcup_{i=1}^{m} g_{i}(\partial A)$ is empty.

After a general position argument, we may suppose that $h_{0}^{-1}\left(\bigcup_{i=1}^{m} g_{i}(\partial A)\right)$ is a finite set. We assume also that there is no map $h_{1}\left(A_{j}, \partial A_{j}\right) \rightarrow\left(M, F_{1}\right)$ homotopic to $h_{0}:\left(A_{j}, \partial A_{j}\right) \rightarrow\left(M, F_{1}\right)$ such that $h_{1}^{-1}\left(\bigcup_{i=1}^{m} g_{i}(\partial A)\right)$ contains fewer points than $h_{0}^{-1}\left(\bigcup_{i=1}^{m} g_{i}(\partial A)\right)$. After a general position argument, we may assume that $h_{0}^{-1}\left(\bigcup_{i=1}^{m} g_{i}(A)\right)=J$ is a collection of disjoint simple arcs and loops properly embedded in $A_{j}$. We assume also that if $h_{1}$ is homotopic to $h_{0} \operatorname{rel} \partial A_{j}$, $h_{1}^{-1}\left(\bigcup_{i=1}^{m} g_{i}(A)\right)$ contains no fewer components than $J$. 
Suppose that $\lambda \subset J$ is a nullhomeotopic simple loop on $A_{j}$. Since $h_{0}(\lambda)$ is nullhomotopic in $\bigcup_{i=1}^{m} g_{i}(A)$, we can modify $h_{0}$ on a neighborhood of the disk bounded by $\lambda$ to obtain a map $h_{1}$, homotopic to $h_{0}$ rel $\partial A_{j}$, that contradicts our choice of $h_{0}$. Suppose there is an arc $\beta_{1} \subset J$ having both its endpoints on a single component of $\partial A_{j}$. Thus there is an $\operatorname{arc} \beta_{2} \subset \partial A_{j}$ such that $\beta_{1} \cup \beta_{2}$ bounds a disk $D$ on $A_{j}$. We observe that $h_{0}\left(\beta_{1}\right)$ is homotopic rel its endpoints to an arc in $\bigcup_{i=1}^{m} g_{i}(\partial A)$ and thus $h_{0}\left(\beta_{2}\right)$ is homotopic rel its endpoints to an arc in $\bigcup_{i=1}^{m} g_{i}(\partial A)$. Thus it can be seen that $h_{0}$ is homotopic to a map $h_{1}$, as above, such that $h_{1}^{-1}\left(\bigcup_{n=1}^{m} g_{i}(\partial A)\right)$ contains fewer points than $h_{0}^{-1}\left(\bigcup_{i=1}^{m} g_{i}(\partial A)\right)$. This contradicts our choice of $h_{0}$ and we conclude that either $h_{0}^{-1}\left(\bigcup_{i=1}^{m} g_{i}(\partial A)\right)$ is empty as was to be shown or $J$ is a collection of disjoint spanning arcs of $A_{j}$. In the latter case it is not difficult to show that after a homotopy we may suppose that $h_{0}(J)$ is a nonempty collection of disjoint simple spanning arcs on some of the $g_{i}(A)$ for $i=1, \ldots, m$.

Suppose $h_{0}(J)$ does not lie on one component of $\bigcup_{i=1}^{m} g_{i}(A)$. Let $D$ be the closure of a component of $A_{j}-J$. We may suppose that $h_{0}(D \cap J)$ does not lie on a single component of $\bigcup_{i=1}^{m} g_{i}(A)$. It is a consequence of the proof of the loop theorem [12] that there is an embedding $h: D \rightarrow M$ such that $h(D) \cap$ $\bigcup_{i=1}^{m} g_{i}(A)=h_{0}(D) \cap \bigcup_{i=1}^{m} g_{i}(A)$ and $h(D) \cap\left(F_{1} \cup \bigcup_{i=1}^{m} g_{i}(A)\right)=h(\partial D)$. We suppose that $h(D) \cap g_{r}(A)$ and $h(D) \cap g_{s}(A)$ are nonempty where $1 \leqslant r<s \leqslant m$. We will show that $g_{r}(A)$ and $g_{s}(A)$ are parallel. We suppose that $h(D)$ is in general position with respect to $\partial M_{1}$. Then $h(D) \cap \partial M_{1}$ may be taken to be a simple loop $\lambda$. If $\lambda$ lies on $F_{2}, \lambda$ is homotopic to a point on $F_{2}$ since $F_{2}$ is incompressible in $M$. It follows that $h(D)$ could be taken to lie in $M_{1}$. This is impossible since the retraction of $M_{1}$ to $F_{1} \cup \cup_{i=1}^{m} g_{i}(A)$ would yield a retraction of $h(D)$ to $h(\partial D)$.

Thus $\lambda$ must be on a toroidal boundary component $T_{1}$ of $M_{1}$. Note that $T_{1}$ is a torus and that $g_{r}(A) \cup g_{s}(A)$ together with two annuli on $F_{1}$ form a compressible torus. Let $U$ be a regular neighborhood of $T_{1} \cup h(D)$. Then one component of $\partial U$ is a 2-sphere; and since $M$ is irreducible, it can be seen that $T_{1}$ bounds a solid torus in $M$. Since this solid torus meets $\partial M_{1}$ only in $T_{1}$, it can be seen that $g_{r}(A)$ and $g_{s}(A)$ are parallel rel $F_{1}$. Thus we may assume that $h_{0}(J)$ lies on $g_{1}(A)$.

Let $\hat{M}_{1}$ be a regular neighborhood of $F_{1} \cup g_{1}(A)$ in $M_{1}$. Let $\hat{F}_{2}$ be a component of $\operatorname{cl}\left(\partial M_{1}-\left(\partial M \cup F_{1}\right)\right)$ that fails to separate $M$. By Lemma 5.2, $\hat{F}_{2}$ is good in $M$, and thus $\hat{F}_{2}$ is incompressible in $M$. Since $\partial M_{1}$ can be obtained by removing two annuli from and adding two annuli to the boundary of a regular neighborhood of $F_{1}$ in $M_{1}, \partial \hat{M}_{1}$ has at most two components if $F_{1}$ has boundary and three components if $F_{1}$ is closed. We suppose that $h_{0}$ is in general position with respect to $\partial \hat{M}_{1}$. Then $h_{0}^{-1}\left(\hat{F}_{2}\right)$ is a nonempty collection of simple loops in 
the complement of $J$. Let $D$ be the closure of a component of $A_{j}-J$ that meets $h_{0}^{-1}\left(\hat{F}_{2}\right)$. It can be seen that $D \cap h_{0}^{-1}\left(\hat{F}_{2}\right)$ is a single simple loop $\lambda$. Since $\hat{F}_{2}$ is incompressible $h_{0}(\lambda)$ is nullhomotopic on $\hat{F}_{2}$. It follows that there is a map $h_{1}$ of $A_{j}$ into $M$ such that $h_{1}(\tau) \subset \hat{F}_{2}$ and $h_{1}\left|\left(A_{j}-D\right)=h_{0}\right|\left(A_{j}-D\right)$. Thus after a homotopy rel $\partial A_{j}$, we may suppose that $h_{0}(D) \subset \hat{F}_{2}$ since $\pi_{2}(M)=0$.

Let $h=h_{0} \mid D$. Since there is a deformation of $\hat{M}_{1}$ onto $F_{1} \cup g_{1}(A), h$ is homotopic rel $\partial D\left(J \cup \partial A_{j}\right.$, if $J$ is connected) to a map $\hat{h}$ into $F_{1} \cup g_{1}(A)$. It follows from standard arguments that $\hat{h}$ is homotopic rel $\partial D$ to a map $\hat{h}_{1}: D \rightarrow$ $F_{1} \cup g_{1}(A)$ such that $\hat{h}_{1}^{-1}\left(g_{1}(\partial A)\right)$ is a collection of disjoint simple arcs properly embedded in $D$.

Suppose $\beta_{1}$ is a simple arc in $\hat{h}_{1}^{-1}\left(g_{1}(\partial A)\right)$ properly embedded in $D$. Then $\beta_{1}$ cuts off a disk $D_{1}$ on $D$ such that $D_{1} \cap \hat{h}_{1}^{-1}\left(g_{1}(\partial A)\right)=\beta_{1}$ for $\partial D \cap \hat{h}_{1}^{-1}\left(g_{1}(\partial A)\right)$ contains exactly four (or two) points. Let $\beta_{2}$ be the closure of $\partial D_{1}-\beta_{1}$. Then $\hat{h}_{1}\left(\beta_{2}\right)$ is homotopic rel its endpoints to $\hat{h}_{1}\left(\beta_{1}\right)$. But $\beta_{2} \subset \partial A_{j}$ and $h_{0}\left(\beta_{2}\right)$ is now seen to be homotopic rel its endpoints to an arc on $g_{1}(\partial A)$. This contradicts our choice of $h_{0}$.

Our claim follows and we suppose that $f^{-1}\left(\bigcup_{i=1}^{m} g_{i}(\partial A)\right) \cap A_{j}$ is empty for each $j$ such that $\bigcup_{i=1}^{m} g_{i}(A) \cup f\left(A_{j}\right)$ meets only one component of $N-F_{1}$ where $1 \leqslant j \leqslant n$.

Suppose that there is an integer $j$ where $1 \leqslant j \leqslant n$ such that $f\left(\alpha_{j}\right)$ meets both components of $N-F_{1}$. We claim that there is a map $f_{1}$ homotopic to $f$ under a homotopy constant outside of a neighborhood of $A_{j}$ such that $A_{j} \cap$ $f_{1}^{-1}\left(\bigcup_{i=1}^{m} g_{i}(A)\right)$ is a collection (possibly empty) of disjoint simple essential loops and $f_{1}^{-1}\left(\bigcup_{i=1}^{m} g_{i}(A)\right) \subseteq f^{-1}\left(\bigcup_{i=1}^{m} g_{i}(A)\right)$.

After a general position argument, we may suppose that $f^{-1}\left(\bigcup_{i=1}^{m} g_{i}(A)\right) \cap$ $A_{i}=J$ is the union of a collection of disjoint simple arcs and loops properly embedded in $A_{j}$. After a homotopy we also assume that $f$ has been chosen so that if $f_{1}$ is homotopic to $f$ as above, the number of points in $f^{-1}\left(\bigcup_{i=1}^{m} g_{i}(\partial A)\right) \cap A_{j}$ is no greater than that in $f_{1}^{-1}\left(\bigcup_{i=1}^{m} g_{i}(\partial A)\right) \cap A_{j}$, and the number of components in $f_{1}^{-1}\left(\bigcup_{i=1}^{m} g_{i}(A)\right) \cap A_{j}$ is not less than the number in $J$.

Suppose that $\lambda \subset J$ is a simple nullhomotopic loop. Then as in the proof above we can modify $f$ to find a map $f_{1}$ homotopic to $f$ such that the number of components in $f_{1}^{-1}\left(\bigcup_{i=1}^{m} g_{i}(A)\right) \cap A_{j}$ is less than the number in $J$. Since this contradicts our choice of $f$, we suppose that each simple loop in $J$ is essential.

Suppose that $\beta_{1}$ is an arc in $J$. Since $f\left(\beta_{1}\right)$ lies on one of the $g_{i}(A)$ for $i=1, \ldots, m, f\left(\beta_{1}\right)$ meets only one component of $N-F_{1}$. It follows that $\partial \beta_{1}$ lies on a single component of $\partial A_{j}$. Thus $\beta_{1}$ cuts off a disk $D$ on $A_{j}$. Let $\beta_{2}=$ $\partial D \cap \partial A_{j}$. Since $f\left(\beta_{2}\right)$ is homotopic rel its boundary to an arc in $\bigcup_{i=1}^{m} g_{i}(\partial A)$, it follows as above that there is a map $f_{1}$ homotopic to $f$ such that $f_{1}^{-1}\left(F_{1}\right)=$ $f^{-1}\left(F_{1}\right)$ and $f_{1}^{-1}\left(\bigcup_{i=1}^{m} g_{i}(\partial A)\right) \cap A_{j}$ contains fewer points than does 
$f^{-1}\left(\bigcup_{i=1}^{m} g_{i}(\partial A) \cap A_{j}\right)$. Since this homotopy can be taken to be constant on the complement of a regular neighborhood of $A_{j}$, the existence of $f_{1}$ contradicts our choice of $f$. This establishes our claim.

Suppose $\bar{f}:(A, \partial A) \rightarrow\left(M, F_{1}\right)$ is a map such that $\bar{f}(A) \cap \bigcup_{i=1}^{m} g_{i}(A)$ is empty and $\bar{f}(A) \cup \cup_{i=1}^{m} g_{i}(A)$ meets only one component of $R-F_{1}$. Let $F^{*}$ be the closure of the complement in $F_{1}$ of a regular neighborhood in $F_{1}$ of $\bigcup_{i=1}^{m} g_{i}(\partial A)$. Suppose that $\left.\overline{f(} c_{1}\right)$ is not freely homotopic in $F^{*}$ to a loop in $\partial F^{*}$, the component of $F^{*}$ on which $\bar{f}\left(c_{1}\right)$ lies is not the planar surface with three boundary components, and $\bar{f}(\alpha)$ is not homotopic rel its endpoints to an arc on $F_{1}$. Then we claim the collection $g_{i}$ for $i=1, \ldots, m$ is not maximal.

If $\bar{f}\left(c_{1}\right)$ and $\bar{f}\left(c_{2}\right)$ lie on distinct components of $F^{*}$, there is an embedding $g_{m+1}:(A, \partial A) \rightarrow\left(M, F_{1}\right)$ such that $\bigcup_{i=1}^{m+1} g_{i}(A)$ meets only one component of $R-F_{1}, g_{m+1 *}: \pi_{1}(A) \rightarrow \pi_{1}(M)$ is monic, and $g_{m+1}\left(c_{1}\right)$ is not freely homotopic in $F^{*}$ to a component of $\partial F^{*}$ by Theorem 3 . If $g_{m+1}(\alpha)$ is homotopic rel its endpoints to an arc in $F^{*}, g_{m+1}$ is homotopic rel $\partial A$ to a map into $F_{1}$ by Proposition 3.2 since $\pi_{2}(M)=0$ and $F_{1}$ is incompressible. But then $g_{m+1}\left(c_{1}\right)$ is freely homotopic in $F_{1}$ to $g_{m+1}\left(c_{2}\right)$ and it follows from standard arguments that $g_{m+1}$ is homotopic in $F^{*}$ to a loop in $\partial F^{*}$ since each loop in $\partial F^{*}$ is essential in $F_{1}$. Clearly $g_{m+1}(A)$ is not parallel to $g_{i}(A)$ for $1 \leqslant i \leqslant m$ since the loop $g_{m+1}\left(c_{1}\right)$ is not freely homotopic to a loop in $\partial F^{*}$. Thus $g_{m+1}$ extends the collection $\left\{g_{i}: 1 \leqslant i \leqslant m\right\}$ and we suppose that $\bar{f}\left(c_{1}\right)$ and $\bar{f}\left(c_{2}\right)$ lie on a single component of $F^{*}$.

We may suppose that $\bar{f}^{-1}\left(\partial M_{1}-F_{1}\right)$ is the union of two essential simple loops since $\bar{f}(A) \cap \bigcup_{i=1}^{m} g_{i}(A)$ is empty. Since the component of $F^{*}$ on which $\bar{f}\left(c_{1} \cup c_{2}\right)$ lies is not an annulus, $\bar{f}\left(\bar{f}^{-1}\left(\partial M_{1}-F_{1}\right)\right)$ lies on $F_{2}$. Now $\bar{f}$ induces a map $\bar{f}_{1}:(A, \partial A) \rightarrow\left(M, F_{2}\right)$ by restriction of $\bar{f}$ to the closure of the central component of $A-\bar{f}^{-1}\left(F_{2}\right)$. Suppose $\bar{f}_{1}(\alpha)$ is homotopic rel its endpoints to an arc on $F_{2}$. Since $F_{2}$ is incompressible and $\pi_{2}(M)=0, \bar{f}_{1}$ and $\bar{f}$ are homotopic rel $\partial A$ to a map into $M_{1}$. Since there is a deformation retraction of $M_{1}$ to $F_{1} \cup \bigcup_{i=1}^{m} g_{i}(A), \bar{f}$ is homotopic rel $\partial A$ to a map into $F_{1} \cup \bigcup_{i=1}^{m} g_{i}(A)$ and $\bar{f}\left(c_{1}\right)$ is homotopic in $F^{*}$ to a loop in $\partial F^{*}$.

Thus we may assume $\bar{f}_{1}\left(\alpha_{1}\right)$ is not homotopic rel its endpoints to a map into $F_{2}$. It is now a consequence of Theorem 2 that there is an $F_{2}$-essential embedding $g:(A, \partial A) \rightarrow\left(M, F_{2}\right)$ such that

(1) $g(\partial A)$ lies in a regular neighborhood in $F_{2}$ of $\bar{f}_{1}(\partial A)$.

(2) $g^{-1}\left(M_{1}\right)=\partial A$.

We wish to replace $g$ by a map $g_{m+1}:(A, \partial A) \rightarrow\left(M, F_{1}\right)$ that extends the collection of $g_{i}$ for $i=1, \ldots, m$. We observe that there are disjoint simple loops $\lambda_{1}$ and $\lambda_{2}$ on the component of $F^{*}$ on which $\left.\overline{f(} c_{1} \cup c_{2}\right)$ lies such that $\lambda_{j} \cup g\left(c_{j}\right)$ bounds an embedded annulus for $j=1,2$ because of the local product structure 
between $F^{*}$ and portions of $\partial M_{1}$. After the usual argument we may assume that these annuli are disjoint. Let $g_{m+1}:(A, \partial A) \rightarrow\left(M, F_{1}\right)$ be an embedding such that $g_{m+1}(A)$ is the union of $g(A)$ and the two annuli mentioned above.

Clearly $g_{m+1 *}: \pi_{1}(A) \rightarrow \pi_{1}(M)$ is monic. If $g_{m+1}(\alpha)$ is homotopic rel its endpoints to an arc on $F_{1}, g_{m+1}$ is homotopic rel $\partial A$ to a map into $F_{1}$ by Proposition 3.2 since $F_{1}$ is incompressible and $\pi_{2}(M)=0$. Suppose that $H: A \times$ $[0,1] \rightarrow M$ is such a homotopy. Using standard techniques we may assume $H^{-1}\left(F_{2}\right)$ is an incompressible surface properly embedded in $A \times[0,1]$. Since $H^{-1}\left(F_{2}\right) \cap \partial(A \times[0,1])$ is a pair of disjoint essential loops, $H^{-1}\left(F_{2}\right)$ is an embedded annulus. By Proposition 3.1 in [1] this annulus is parallel to an annulus in $\partial(A \times[0,1])$ and it can be seen that $g$ must have been homotopic rel $\partial A$ to a map into $F_{2}$. Thus we suppose that $g_{m+1}(\alpha)$ is not homotopic rel its endpoints to an arc in $F_{1}$.

If $g_{m+1}(A)$ is parallel rel $F_{1}$ to $g_{j}(A)$ where $1 \leqslant j \leqslant m$, there is an embed$\operatorname{ding} H: A \times[0,1] \rightarrow M$ such that

(1) $H(A \times\{0\})=g_{m+1}(A)$.

(2) $H(A \times\{1\})=g_{j}(A)$ for some $j$ where $1 \leqslant j \leqslant m$.

(3) $H(\partial A \times[0,1]) \subset F_{1}$.

But then $H^{-1}\left(F_{2}\right)$ is an annulus embedded in $A \times[0,1]$, and it can be seen as above that $g(A)$ is homotopic to a map into $F_{2}$. It follows that $g_{m+1}:(A, \partial A)$ $\rightarrow\left(M, F_{1}\right)$ extends the collection of $g_{i}$ for $i=1, \ldots, m$, and the proof of our claim is complete.

Let $F_{3}$ be the closure of $\operatorname{int}(M) \cap \partial M_{2}-F_{1}$. Let $g_{i}^{\prime}:(A, \partial A) \rightarrow\left(M, F_{3}\right)$ for $i=1, \ldots, m^{\prime}$ be a maximal collection of disjoint $F_{3}$ essential embeddings such that

(1) $g_{i}^{\prime}(A) \cap\left(M_{2}-F_{3}\right)$ is empty for $i=1, \ldots, m^{\prime}$.

(2) $g_{i}^{\prime}(A)$ and $g_{j}^{\prime}(\alpha)$ are not parallel rel $F_{3}$ for $1 \leqslant i<j \leqslant m^{\prime}$.

After an argument similar to the one given above we may assume

(1) $f^{-1}\left(F_{1} \cup F_{3}\right)$ is a collection of simple essential loops,

(2) $f\left(K_{1}\right) \cap\left(\bigcup_{i=1}^{m} g_{i}(\partial A) \cup \bigcup_{i=1}^{m^{\prime}} g_{i}^{\prime}(\partial A)\right)$ is empty.

(3) $f^{-1}\left(\bigcup_{i=1}^{m} g_{i}(A)\right)$ and $f^{-1} \bigcup_{i=1}^{m} g_{i}^{\prime}(A)$ are unions of nonempty collections of disjoint simple essential loops.

(4) There is no map $f_{1}$ homotopic to $f$ such that $f_{1}^{-1}\left(F_{1} \cup F_{3}\right)$ contains fewer loops than does $f^{-1}\left(F_{1} \cup F_{3}\right)$.

We now let $A_{1}, \ldots, A_{n}$ be the closures of the components of $K_{1}-$ $f^{-1}\left(F_{1} \cup F_{3}\right)$. Let $\bar{F}_{1}\left(\bar{F}_{3}\right)$ be the closure of the complement of a regular neighborhood of $\bigcup_{i=1}^{m} g_{i}(\partial A)\left(\bigcup_{i=1}^{m^{\prime}} g_{i}^{\prime}(\partial A)\right)$ in $F_{1}\left(F_{3}\right)$. We may suppose that $f\left(K_{1}\right) \cap F_{1} \subset \bar{F}_{1}$ and $f\left(K_{1}\right) \cap F_{3} \subset \bar{F}_{3}$. If $f\left(A_{j}\right) \subset M_{2}$, we may suppose that $f$ carries one component of $\partial A_{j}$ to $\bar{F}_{1}$ and the other to $\bar{F}_{3}$ for $1 \leqslant j \leqslant n$. Let $\lambda_{1}, \ldots, \lambda_{n}$ be the components of $f^{-1}\left(F_{1} \cup F_{3}\right)$. We may suppose that $A_{i} \cap$ 
$A_{i+1}=\lambda_{i}$ for $i=1, \ldots, n-1$ and $A_{n} \cap A_{1}=\lambda_{n}$. Now if $\lambda_{i-1}$ and $\lambda_{i}$ are the components of $\partial A_{i}$ and $f\left(\lambda_{i-1} \cup \lambda_{i}\right)$ lies on $\bar{F}_{1}$, it follows from the claim above that $f\left(\lambda_{i-1}\right)\left(f\left(\lambda_{i}\right)\right)$ is freely homotopic in $\bar{F}_{1}$ to a loop in $\partial \bar{F}_{1}$ or the component of $\bar{F}_{1}$ on which $f\left(\lambda_{i-1}\right)\left(f\left(\lambda_{i}\right)\right)$ lies is planar with three boundary components. The statement above is also true if we replace $\bar{F}_{1}$ by $\bar{F}_{3}$.

Let $K$ be a closed surface of genus one of unspecified orientability. We claim there is an essential map $\bar{f}: K \rightarrow M$ such that $\bar{f}^{-1}\left(F_{1} \cup F_{3}\right)$ is a nonempty collection of simple essential loops, $\bar{f} \mid \bar{f}^{-1}\left(\left(F_{1}-\bar{F}_{1}\right) \cup\left(F_{3}-\bar{F}_{3}\right)\right)$ is a homeomorphism, and the restriction of $\bar{f}$ to each component of $K-\bar{f}^{-1}\left(F_{1} \cup F_{3}\right)$ is a homeomorphism. We will construct this map by finding a sequence of $\left(F_{1} \cup F_{3}\right)$ essential embeddings $\bar{f}_{i}:(A, \partial A) \rightarrow\left(M,\left(F_{1}-\bar{F}_{1}\right) \cup\left(F_{3}-\bar{F}_{3}\right)\right)$ for $1 \leqslant i$ such that

(1) $\bar{f}_{i}\left(c_{2}\right)=\bar{f}_{i+1}\left(c_{1}\right)$ for $1 \leqslant i$.

(2) $\bar{f}_{i}\left(c_{1}\right)$ is not freely homotopic to a loop in $\partial M$ for $0<i$ and fitting together a finite sequence of these maps to form $\bar{f}$.

We may suppose that $f\left(\lambda_{1} \cup \lambda_{n}\right) \subset \bar{F}_{1}$ and that for $1 \leqslant j \leqslant n$ the image under $f$ of $\lambda_{j}$ is a simple loop whenever $f\left(\lambda_{j}\right)$ is freely homotopic in $\bar{F}_{1} \cup \bar{F}_{3}$ to a loop in $\partial\left(\bar{F}_{1} \cup \bar{F}_{3}\right)$. Then there is an embedding $\bar{f}_{1}:(A, \partial A) \rightarrow\left(M, F_{1}\right)$ such that $\bar{f}_{1}^{-1}\left(M_{2}\right)=\partial A, \bar{f}_{1}(\alpha)$ is not homotopic rel its endpoints to an arc on $F_{1}$, and $\bar{f}_{1 *}: \pi_{1}(A) \rightarrow \pi_{1}(M)$ is monic by Theorem 2 or Lemma 5.9. If $f\left(\lambda_{1}\right)\left(f\left(\lambda_{n}\right)\right)$ is not freely homotopic in $\bar{F}_{1}$ to a loop in $\partial \bar{F}_{1}$, the component of $\bar{F}_{1}$ on which $f\left(\lambda_{1}\right)\left(f\left(\lambda_{n}\right)\right)$ lies is a planar surface with three boundary components by the claim above which allows us to extend the collection $\left\{g_{i}: i=1, \ldots, m\right\}$; so we may suppose that $\bar{f}_{1}\left(c_{1} \cup c_{2}\right)$ lies in $F_{1}-\bar{F}_{1}$. Since $F_{1}$ is not the planar surface with three boundary components and no loop essential in $\bar{F}_{1}$ is inessential in $F_{1}$, we may suppose that $\bar{f}\left(c_{1}\right)$ is not freely homotopic to a loop in $\partial F_{1}$. Thus since $F_{1}$ is good, $\bar{f}_{1}\left(c_{1}\right)$ is not freely homotopic to a loop in $\partial M$. The reader should notice the trick that allows us to assume that $\bar{f}_{1}\left(c_{1} \cup c_{2}\right) \subset F_{1}-\bar{F}_{1}$ as it will be used again below without further justification. We shall say that $\bar{f}_{1}$ is constructed using $f \mid A_{1}$. Since a regular neighborhood of $\lambda_{i}$ for $i=1, \ldots, n$ meets both $f^{-1}$ (int $\left.\left(M_{2}\right)\right)$ and $f^{-1}\left(M-M_{2}\right)$, there is a smallest integer $j$ such that $f\left(\lambda_{j}\right) \cup f\left(\lambda_{j+1}\right)$ lies on $\bar{F}_{3}$. Let $\bar{A}=\bigcup_{i=2}^{\prime} A_{i}$ and $g=f \mid \bar{A}$. Let $(\widetilde{M}, p)$ be the infinite cyclic covering of $M$ associated with $F_{1}$. Let $\widetilde{g}: \bar{A} \rightarrow \widetilde{M}$ be a map such that $p \tilde{g}=g$. Then it can be seen that if $\widetilde{F}$ is a component of $p^{-1}\left(F_{1} \cup F_{3}\right)$, $\widetilde{g}^{-1}(\widetilde{F})$ contains at most a single simple loop. Let $\widetilde{F}_{1}$ and $\widetilde{F}_{3}$ respectively be the components of $p^{-1}\left(F_{1} \cup F_{3}\right)$ on which $\widetilde{g}\left(\lambda_{1}\right)$ and $\widetilde{g}\left(\lambda_{j}\right)$ lie. Let $\widetilde{M}_{1}$ be the closure of the component of $\widetilde{M}-\left(\widetilde{F}_{1} \cup \widetilde{F}_{3}\right)$ in which $\widetilde{g}(\operatorname{int}(\bar{A}))$ lies.

It is a consequence of Theorem 3 or the theorem in [14] that there is an embedding $\hat{g}: \bar{A} \rightarrow \widetilde{M}_{1}$ such that

(1) $\hat{g}_{*}: \pi_{1}(\bar{A}) \rightarrow \pi_{1}\left(\widetilde{M}_{1}\right)$ is monic. 
(2) $p \hat{g}\left(\lambda_{1}\right)=\bar{f}_{1}\left(c_{2}\right)$.

(3) $p \hat{g}\left(\lambda_{j}\right) \subset F_{3}-\bar{F}_{3}$.

(4) $\hat{g}^{-1} p^{-1}\left(F_{1} \cup F_{3}\right)$ is a collection of essential simple loops.

(5) $p \hat{g}\left(\lambda_{i}\right)$ lies in a regular neighborhood in $F_{1} \cup F_{3}$ of $f\left(\lambda_{i}\right)$ for $i=2$, ..., $j-1$.

It is a consequence of (5) above that we may suppose $p \hat{g}(A) \cap\left(\left(F_{1}-\bar{F}_{1}\right)\right.$ $\left.\cup\left(F_{3}-\bar{F}_{3}\right)\right)=p \hat{g}(\partial \bar{A})$. We next apply the theorem [14] to the map $p \hat{g}$ to find an embedding $h: A \rightarrow M$ such that $h(\partial A)=p \hat{g}(\partial \bar{A})$. After the usual argument we may suppose that

(1) $h^{-1}\left(F_{1} \cup F_{3}\right)$ is a collection of simple essential loops.

(2) $h^{-1}\left(\left(F_{1}-\bar{F}_{1}\right) \cup\left(F_{3}-\bar{F}_{3}\right)\right)=\partial A$.

(3) There does not exist a map $h_{1}$ homotopic to $h$ rel $\partial A$ such that $h_{1}^{-1}\left(F_{1} \cup F_{3}\right)$ contains fewer loops than $h^{-1}\left(F_{1} \cup F_{3}\right)$.

Let $\bar{f}_{2}=h$.

We next apply Lemma 5.9 or Theorem 2 to $f \mid A_{j+1}$ to find an embedding $\bar{f}_{3}^{\prime}:(A, \partial A) \rightarrow\left(M, F_{3}\right)$ such that

(1) $\bar{f}_{3}^{\prime}\left(c_{1}\right)=\bar{f}_{2}\left(c_{2}\right)$.

(2) $\bar{f}_{3}^{\prime}(\alpha)$ is not homotopic rel its endpoints to an arc in $F_{3}$.

(3) $\bar{f}_{3}^{\prime}\left(c_{2}\right)$ lies in a regular neighborhood in $F_{3}$ of $f\left(\lambda_{j+1}\right)$.

Since $\bar{f}_{3}^{\prime}\left(c_{2}\right)$ is simple, we may replace $\bar{f}_{3}^{\prime}$ by an embedding $\bar{f}_{3}$ satisfying (1) and (2) above such that $\bar{f}_{3}\left(c_{2}\right)$ lies on $F_{3}-\bar{F}_{3}$.

Suppose we have constructed $\bar{f}_{i}$ and $\bar{f}_{i+1}$. We then use the same technique to construct $\bar{f}_{i+2}$ as that used to construct $\bar{f}_{i}$ except that after we use $f \mid A_{n}$ to construct some $\bar{f}_{k}$, we will need to use $f \mid A_{1}$ to construct $\bar{f}_{k+1}$. Similarly after we reuse $f \mid A_{1}$ to construct some $\bar{f}_{k}$, we must reuse $f \mid \bar{A}$ to construct $\bar{f}_{k+1}$, etc. Note that $\bar{f}_{i}\left(c_{1}\right)$ and $\bar{f}_{i}\left(c_{2}\right)$ are simple loops freely homotopic in the closure of $\left(F_{1}-\bar{F}_{1} \cup F_{3}-\bar{F}_{3}\right)$ to a component of $\partial\left(\bar{F}_{1} \cup \bar{F}_{3}\right)$. We suppose $\bar{f}_{1}, \bar{f}_{1+k}$, $\bar{f}_{1+2 k}$, and $\bar{f}_{1+3 k}$ are constructed using $f \mid A_{1}$. Since $f\left(\lambda_{n}\right)$ is freely homotopic in $\bar{F}_{1}$ to a loop on $\partial \bar{F}_{1}$ or the component of $\bar{F}_{1}$ on which $f\left(\lambda_{n}\right)$ lies is a planar surface with three boundary components, some pair of loops among $\bar{f}_{1}\left(c_{1}\right)$, $\bar{f}_{1+k}\left(c_{1}\right), \bar{f}_{1+2 k}\left(c_{1}\right)$, and $\bar{f}_{1+3 k}\left(c_{1}\right)$ must be freely homotopic. It will be seen below that which pair is freely homotopic is not relevant so we may suppose for our convenience that $\bar{f}_{1}\left(c_{1}\right)$ and $\bar{f}_{k+1}\left(c_{1}\right)$ are freely homotopic and, after a homotopy, $\bar{f}_{k}\left(c_{2}\right)=\bar{f}_{1}\left(c_{1}\right)$. We suppose that $K$ is the union of annuli $\bar{A}_{1}, \ldots, \bar{A}_{k}$ such that $\bar{A}_{i} \cap \bar{A}_{i+1}=\bar{\lambda}_{i}$ is a component of $\partial \bar{A}_{i}$ for $i=1, \ldots, k-1$ and $\bar{A}_{k} \cap \bar{A}_{1}=\bar{\lambda}_{k}$. We define $\bar{f}: K \rightarrow M$ by $\bar{f}\left(\bar{A}_{i}\right)=\bar{f}_{i}(A)$ for $1 \leqslant i \leqslant k$ so that $\bar{f}$ will be continuous and note that any collection of embeddings as the $\bar{f}_{i}$ determine a singular map as $\bar{f}$. After a small motion of $\bar{f}$, we may suppose that $\bar{f} \mid \bar{f}^{-1}\left(\left(F_{1}-\bar{F}_{1}\right) \cup\left(F_{3}-\bar{F}_{3}\right)\right)$ is a homeomorphism.

We wish to show that $\bar{f}$ is essential. We observe that $\bar{f}\left(\bar{\lambda}_{j}\right)$ is freely homo- 
topic to $\bar{f}_{i}\left(c_{1}\right)$ for $1 \leqslant i$ and $1 \leqslant j \leqslant k$ so the loop $\bar{f}\left(\bar{\lambda}_{j}\right)$ is not freely homotopic to a loop on $\partial M$ since $F_{1}$ is good. It is a consequence of Lemma 5.4 that $\bar{f}_{*}$ : $\pi_{1}(K) \rightarrow \pi_{1}(M)$ is monic since $\bar{f}_{1 *}: \pi_{1}(A) \rightarrow \pi_{1}(M)$ is monic. Thus $\bar{f}$ is essential and our claim is established.

We complete the proof of Lemma 5.12 by applying Lemma 5.13 below.

LEMMA 5.13. Let $F$ be a good surface in $M, K$ a closed, connected surface of genus one, and $f: K \rightarrow M$ an essential map such that

(1) $f^{-1}(F)$ is the union of a collection of disjoint simple essential loops $\lambda_{1}, \lambda_{2}, \ldots, \lambda_{n}$ where $n \geqslant 2$.

(2) $f\left(\lambda_{1}\right) \cap \bigcup_{i=2}^{n} f\left(\lambda_{i}\right)$ is empty.

(3) $f \mid \lambda_{i}$ is a homeomorphism for $i=1, \ldots, n$ and $f\left(\lambda_{1}\right)$ is not freely homotopic on $F$ to a loop in $\partial F$.

(4) The closures of the components of $K-\bigcup_{i=1}^{n} \lambda_{i}$ are annuli $A_{1}, \ldots, A_{n}$.

(5) $f \mid \operatorname{int}\left(A_{i}\right)$ is a homeomorphism.

(6) $f:\left(A_{i}, \partial A_{i}\right) \rightarrow(M, F)$ is an F-essential map.

Then there is a closed connected surface $K_{1}$ of genus one and an essential embedding $g: K_{1} \rightarrow M$ such that $g^{-1}(F)$ is a nonempty collection of essential loops $\lambda_{1}^{*}, \ldots, \lambda_{m}^{*}$ and $g\left(\lambda_{1}^{*}\right)$ is not freely homotopic on $F$ to a loop in $\partial F$.

Proof. Let $X(f)=\bigcup_{i \neq j ; 1 \leqslant i<j \leqslant n}\left(f\left(\lambda_{i}\right) \cap f\left(\lambda_{j}\right)\right)$. We assume that $f$ has been chosen so that

(a) $f$ satisfies conditions (1) $-(6)$ above.

(b) $f\left(\lambda_{i}\right) \cap f\left(\lambda_{j}\right) \cap f\left(\lambda_{k}\right)$ is empty if $1 \leqslant i<j<k \leqslant n$.

(c) if $f_{1}$ is homotopic to $f$ and satisfies conditions (a) and (b) above, the cardinality of $X(f)$ is less than or equal to that of $X\left(f_{1}\right)$.

It follows from standard arguments that $X(f)$ is a finite set. We claim $X(f)$ is empty.

After a homotopy that keeps $f^{-1}(F)$ fixed we may suppose that $\operatorname{cl}\left(f^{-1} f\left(A_{j}\right) \cap\right.$ int $\left.A_{i}\right)$ is the union of collection of disjoint simple arcs and loops properly embedded in $A_{i}$ where $1 \leqslant i, j \leqslant n$ and $i \neq j$. Suppose that $X(f)$ is not empty. Since $f\left(\lambda_{1}\right) \cap X(f)$ is empty there are integers $i$ and $j$ where $i \neq j$ such that $\operatorname{cl}\left(f^{-1} f\left(A_{i}\right) \cap \operatorname{int}\left(A_{j}\right)\right)$ contains an arc $\beta_{1}$ having both its endpoints on a single component of $\partial A_{j}$. Now $\beta_{1}$ cuts off a disk $D_{1}$ on $A_{j}$. Note that $f \mid D_{1}$ is an embedding. After the usual arguments, we may suppose that $D_{1} \cap$ $f^{-1} f \operatorname{int}\left(A_{i}\right)=\operatorname{int}\left(\beta_{1}\right)$. Let $\beta_{2}$ be the arc on $A_{i}$ such that $f\left(\beta_{1}\right)=f\left(\beta_{2}\right)$. Now $\beta_{2}$ cuts off a disk $D_{2}$ on $A_{i}$ and $f\left(D_{1}\right) \cup f\left(D_{2}\right)$ is a disk embedded in $M$. Let $\gamma_{1}=\operatorname{cl}\left(\partial D_{1}-\beta_{1}\right)$ and $\gamma_{2}=\operatorname{cl}\left(\partial D_{2}-\beta_{2}\right)$. Since $F$ is incompressible in $M, f\left(\gamma_{1}\right)$ $\cup f\left(\gamma_{2}\right)$ bounds a disk $D$ on $F$. After the usual argument, we may suppose that each arc in $f\left(\lambda_{i}\right)$ for $i=1, \ldots, n$ properly embedded in $D$ meets both $f\left(\gamma_{1}\right)$ and $f\left(\gamma_{2}\right)$. But it can now be seen that $f$ was not chosen so that $X(f)$ contains a 
minimal number of points since there is a homotopy $f_{t}: K \rightarrow M$ such that $f_{0}=$ $f, f_{t}^{-1}(F)=\bigcup_{i=1}^{n} \lambda_{i}$ for $t \in[0,1]$, and the cardinality of $X\left(f_{1}\right)$ is less than that of $X(f)$. This establishes our claim.

We continue the proof of Lemma 5.13 with the added assumption that $f \mid f^{-1}(F)$ is a homeomorphism. We suppose that $f$ has been chosen subject to the further restraint that $f^{-1}(F)$ contains as few essential loops as possible but at least one loop. Let $R$ be a regular neighborhood of $F$ and $F_{1}$ and $F_{2}$ the closures of the components of $\mathrm{bd}(R)-\mathrm{bd}(M)$. We may suppose that $f \mid f^{-1}(R)$ is a homeomorphism. Let $B_{1}, \ldots, B_{n}$ be the closures of the components of $K-f^{-1}(R)$. Note that $f \mid B_{i}$ is a homeomorphism for $i=1, \ldots, n$.

Suppose that $i \neq j, f\left(\partial B_{i}\right) \cup f\left(\partial B_{j}\right)$ bounds disjoint annuli $\bar{A}_{1}$ and $\bar{A}_{2}$ in $F_{1} \cup F_{2}$, and $f\left(\partial B_{i}\right)$ meets both $\partial \bar{A}_{1}$ and $\partial \bar{A}_{2}$. Then we can find $\left(F_{1} \cup F_{3}\right)$ essential embeddings $g_{1}, g_{2}:(A, \partial A) \rightarrow\left(\mathrm{cl}(M-R), f\left(\partial B_{i} \cup \partial B_{j}\right)\right)$ that are parallel rel $\left(F_{1} \cup F_{3}\right)$. Note that it is a consequence of Lemma 5.3 that $f\left(K-\left(B_{i} \cup B_{j}\right)\right) \cup g_{1}(A) \cup g_{2}(A)$ determines one or two essential maps of a closed connected surface of genus one. Thus we may suppose that if $B_{i}$ and $B_{j}$ are as above $f\left(B_{i}\right)$ is parallel to $f\left(B_{j}\right)$ rel $\left(F_{1} \cup F_{3}\right)$.

Suppose that $1 \leqslant i<j<k \leqslant n$ and $f\left(\lambda_{i}\right), f\left(\lambda_{j}\right)$ and $f\left(\lambda_{k}\right)$ lie on a single annulus embedded in $F$. Let $f_{1}$ be a map homotopic to $f$ such that $f_{1}\left(\lambda_{i}\right)=$ $f_{1}\left(\lambda_{j}\right)=f_{1}\left(\lambda_{k}\right), f_{1}^{-1}(F)=f^{-1}(F)$, and $f_{1} \mid A_{v}$ is a homeomorphism for $v=1$, $\ldots, n$. Let $A_{1}^{*}, A_{2}^{*}$, and $A_{3}^{*}$ be the closures of the components of $K-$ $\left(\lambda_{i} \cup \lambda_{j} \cup \lambda_{k}\right)$. Then it can be seen that $f_{1} \mid A_{1}^{*}$ and $f_{1} \mid A_{2}^{*}$ or $f_{1} \mid\left(A_{1}^{*} \cup A_{2}^{*}\right)$ determines an essential map $g: K_{1} \rightarrow M$ such that $g^{-1}(F)$ contains fewer loops than does $f^{-1}(F)$; note that such a map will be determined by $f_{1} \mid A_{1}^{*}$ if $f_{1}$ does not carry a regular neighborhood of $\partial A_{1}^{*}$ in $A_{1}^{*}$ into a single component of $R-F$.

It follows that we may suppose that not more than two of the $\lambda_{i}$ are mapped by $f$ into a single annulus on $F$.

Suppose that $f\left(B_{i}\right)$ and $f\left(B_{j}\right)$ are in general position with respect to one another where $i \neq j$ and that $f\left(B_{i}\right) \cap f\left(B_{j}\right)$ contains an essential loop. Let $A_{1}^{*}$ and $A_{2}^{*}$ be the closures of the components of $K-\left(B_{i} \cup B_{j}\right)$. Suppose $f\left(\partial A_{1}^{*}\right)$ meets both $F_{1}$ and $F_{2}$. It can be seen that there is an annulus $B_{1}^{*}$ properly embedded in $M-\operatorname{int}(R)$ such that $\partial B_{1}^{*}=f\left(\partial A_{1}^{*}\right)$. But $B_{1}^{*}$ must be $\left(F_{1} \cup F_{3}\right)$ essential and it is a consequence of Lemma 5.3 that we can construct an essential map $f_{1}: K_{1} \rightarrow M$ such that $f_{1}\left(K_{1}\right)=B_{1}^{*} \cup f\left(A_{1}^{*}\right)$ and $f_{1}^{-1}(F)$ contains fewer loops than $f^{-1}(F)$. This contradicts our construction of $f$.

Thus $f\left(\partial A_{1}^{*}\right)$ may be supposed to lie on $F_{1}$. If the loops in $f\left(\partial A_{1}^{*}\right)$ do not bound an annulus on $F_{1}$, we can find an annulus $B_{1}^{*}$ properly embedded in $M-\operatorname{int}(R)$ such that $\partial B_{1}^{*}=f\left(\partial A_{1}^{*}\right)$. Note that $B_{1}^{*}$ is $F_{1}$ essential since otherwise it is a consequence of Proposition 3.2 that $\partial B_{1}^{*}$ bounds an annulus on $F_{1}$. Now there is an essential map $f_{1}: K_{1} \rightarrow M$ such that $f_{1}\left(K_{1}\right)=B_{1}^{*} \cup f\left(A_{1}^{*}\right)$ and $f_{1}^{-1}(F)$ 
contains fewer loops than $f^{-1}(F)$. Since this contradicts our construction of $F$, we may suppose that $f\left(\partial A_{1}^{*}\right)$ bounds an annulus on $F_{1}$. Similarly $f\left(\partial A_{2}^{*}\right)$ bounds an annulus on $F_{1} \cup F_{2}$. It follows that $f\left(\partial B_{1}\right) \cup f\left(\partial B_{2}\right)$ bounds a pair of disjoint annuli on $F_{1} \cup F_{2}$ and that $f\left(B_{1}\right)$ meets both of these annuli. But then in our construction of $f, f\left(B_{1}\right)$ and $f\left(B_{2}\right)$ were chosen to be parallel rel $\left(F_{1} \cup F_{2}\right)$. This is a contradiction and $f\left(B_{i}\right) \cap f\left(B_{j}\right)$ contains no essential loops if $i \neq j$.

Since $\pi_{2}(M)=0$, it can be shown that there is a map $f_{1}$ homotopic to $f$ rel $R$ such that $f_{1}\left(B_{j}\right) \cap f_{1}\left(B_{i}\right)$ is empty if $1 \leqslant i<j \leqslant n$. Lemma 5.13 follows.

VI. The torus theorem. In this section we state and prove Waldhausen's "torus theorem."

THEOREM 4. Let $M$ be a compact, irreducible 3-manifold with nonvacuous boundary. If $M$ admits an essential map of a torus, $M$ admits an essential embedding of either a torus or an annulus.

Proof. Let $f: T \rightarrow M$ be an essential map. Suppose $D$ is a disk properly embedded in $M$. We suppose that $f$ is in general position with respect to $D$. Then $f^{-1}(D)$ can contain no essential simple loops since $f_{*}: \pi_{1}(T) \rightarrow \pi_{1}(M)$ is monic. It is not difficult to see that $f$ is homotopic to a map $f_{1}: T \rightarrow M$ such that $f_{1}(T) \cap D$ is empty since $\pi_{2}(M)=0$. It follows that we may assume that $\partial M$ is incompressible.

Let $M=M_{1}$ and $M_{i}, F_{i} \subset M_{i}, U\left(F_{i}\right) \subset M_{i}$ for $1 \leqslant i \leqslant n$ be a special hierarchy for $M_{1}$ with respect to $f$. We may suppose that $k$ is an integer, $f(T) \subset M_{k}$, and if $f_{1}$ is any map homotopic to $f$ such that $f_{1}(T) \subset M_{k}, f_{1}(T) \cap F_{k}$ is not empty. We claim that either $M_{k}$ admits an essential map or $f$ is homotopic to a map into $\partial M_{k}$.

Since $f$ may be chosen so that $f(T)$ does not meet any disk $D$ properly embedded in $M_{k}$, we may suppose that $F_{k}$ is not a disk and $\partial M_{k}$ is incompressible in $M_{k}$. Suppose that $f: T \rightarrow M_{k}$ is not essential. Let $\lambda_{1}$ and $\lambda_{2}$ be simple loops on $T$ which meet at a single point and cross at that point. Then $f$ is homotopic in $M_{k}$ to a map $f_{1}$ such that $f_{1}\left(\lambda_{1}\right) \subset \partial M_{k}$. We split $T$ along $\lambda_{1}$ to obtain an annulus $A$. Now $f_{1}$ induces a map $\bar{f}:(A, \partial A) \rightarrow\left(M_{k}, \partial M_{k}\right)$. If $\bar{f}$ is essential, our claim is established. Otherwise by Proposition 3.2, $\bar{f}$ is homotopic rel $\partial A$ to a map into $\partial M_{k}$ since $\partial M_{k}$ is incompressible and $\pi_{2}\left(M_{k}\right)=0$. It follows that $f$ is homotopic to a map into $\partial M_{k}$ and our claim is established.

If $f_{1}: T \rightarrow \partial M_{k}$ is a map homotopic to $f$ and $T_{1}=f_{1}(T), T_{1}$ is a torus since $\partial M_{k}$ is incompressible in $M_{k}, \pi_{1}\left(M_{k}\right) \rightarrow \pi_{1}(M)$ is monic, and $f_{1 *}\left(\pi_{1}(T)\right) \cong$ $Z \oplus Z \subseteq \pi_{1}\left(T_{1}\right)$. Thus it can be seen that $T_{1}$ is incompressible in $M$. Since $f_{1}$ is essential, $T_{1}$ is essential in $M_{1}$ and Theorem 4 follows.

Thus we may suppose that $f: T \rightarrow M_{k}$ is essential in $M_{k}$. It is a consequence of Lemma 5.7 that if $M_{k}$ admits an essential embedding of an annulus or 
a torus, $M_{1}=M$ admits an essential embedding of an annulus or a torus. Thus we may proceed with the added hypothesis that: if $f_{1}$ is any map homotopic to $f$ and $F$ is any good surface in $M, f_{1}(T) \cap F$ is not empty.

If $f$ is $W$-essential, Theorem 4 is a consequence of Lemma 5.4. If $F$ is planar and $\chi(F)=-1$, Theorem 4 is a consequence of Lemma 5.10 .

Let $F_{1}$ be a good surface properly embedded in $M$. After a general position argument, we may suppose that $f^{-1}\left(F_{1}\right)$ is the union of a collection of disjoint simple loops. We assume that $f$ has been chosen so that the number of loops in this collection is minimal. Suppose some loop $\lambda \subset f^{-1}\left(F_{1}\right)$ is inessential. Let the disk $D$ be a regular neighborhood of the disk bounded by $\lambda$ on $T$. Since $F_{1}$ is incompressible, $f(\lambda)$ is inessential on $F_{1}$ and $f(\partial D)$ is nullhomotopic in $M-F_{1}$. Let $f_{1}$ be a map such that $f_{1}|(T-D)=f|(T-D)$ and $f_{1}(D) \cap F_{1}$ is empty. Since $\pi_{2}(M)=0, f_{1}$ is homotopic to $f$. The existence of $f_{1}$ contradicts the minimality condition on $f$ so we may suppose that $f^{-1}\left(F_{1}\right)$ is a collection of disjoint simple essential loops.

Let $A_{1}, \ldots, A_{n}$ be the closures of the components of $T-f^{-1}\left(F_{1}\right)$. Our minimality condition above insures that $f \mid A_{i}$ is not homotopic rel $A_{i} \cap f^{-1}\left(F_{1}\right)$ to a map into $F_{1}$. Let $R$ be a regular neighborhood of $F_{1}$.

Suppose for some $j, f\left(A_{j}\right)$ meets only one component of $R-F_{1}$. It is a consequence of Lemma 5.12 that there is an essential embedding $\bar{f}: K \rightarrow M$ where $K$ is a closed, connected surface of genus one. If $K$ is a torus, we are finished. Otherwise let $N$ be a regular neighborhood of $\bar{f}(K)$. Now $N$ is orientable, so $\partial N$ is a torus and $\pi_{1}(\partial N) \rightarrow \pi_{1}(M)$ is a monic since $\bar{f}_{*}$ is monic. Now $\bar{f}^{-1}\left(F_{1}\right)$ contains a simple loop $\lambda$ such that $\bar{f}(\lambda)$ is not freely homotopic to a loop in $\partial F_{1}$. Since $F_{1}$ is orientable one component of $N \cap F_{1}$ is an annular neighborhood of $\bar{f}(\lambda)$. It follows that $\partial N$ is an essential torus in $M$ since a loop in $\partial N \cap F_{1}$ is not freely homotopic to a loop in $\partial M$.

We suppose for all $i$ where $1 \leqslant i \leqslant n, f\left(A_{i}\right)$ meets both components of $R-F_{1}$ and that $M$ does not admit an essential embedding of $A$. It is a consequence of Lemma 5.11 that there is a surface $K$ such that $\chi(K)=0$ and a map $\overline{f:} K \rightarrow M$ such that

(1) $\bar{f}^{-1}\left(F_{1}\right)$ is a collection of disjoint simple essential loops.

(2) The restriction of $\bar{f}$ to each component of $\bar{f}^{-1}\left(F_{1}\right)$ is a homeomorphism.

(3) The restriction of $\bar{f}$ to each component of $K-\bar{f}^{-1}\left(F_{1}\right)$ is a homeomorphism and the closure of each component of $K-\bar{f}^{-1}\left(F_{1}\right)$ is an annulus. (Note that if $\bar{f}^{-1}\left(F_{1}\right)$ is connected, $\bar{f}$ is an embedding and the proof is completed as above.)

If the image under $\bar{f}$ of some component of $K-\bar{f}^{-1}\left(F_{1}\right)$ meets only one component of $R-F_{1}$, we appeal to Lemma 5.12 and complete the proof of Theorem 4 as above. We suppose that $\bar{f}$ has been chosen so that the images of 
the loops in $\bar{f}^{-1}\left(F_{1}\right)$ are in general position with respect to one another on $F_{1}$. Let $\lambda_{1}, \ldots, \lambda_{n}$ be the components of $\bar{f}^{-1}\left(F_{1}\right)$ and

$$
X(\bar{f})=\bigcup_{i \neq j ; 1<i<j<n}\left(\bar{f}\left(\lambda_{i}\right) \cap \bar{f}\left(\lambda_{j}\right)\right) .
$$

We suppose that $X(\bar{f})$ is a finite set and that if $1 \leqslant i<j<k \leqslant n, \bar{f}\left(\lambda_{i}\right) \cap \bar{f}\left(\lambda_{j}\right) \cap$ $\bar{f}\left(\lambda_{k}\right)$ is empty. Suppose that $\beta_{1}$ and $\beta_{2}$ are arcs embedded in $\bar{f}\left(\lambda_{i}\right)$ and $\bar{f}\left(\lambda_{j}\right)$ respectively such that

(a) $\partial \beta_{1}=\partial \beta_{2}=\beta_{1} \cap \beta_{2}$.

(b) $\beta_{1} \cup \beta_{2}$ bounds a disk $D$ in $F_{1}$.

(c) For each arc $\left.\beta \subset \bigcup_{i=1}^{n} \overline{f(} \lambda_{i}\right)$ properly embedded in $D, \beta$ meets both $\beta_{1}$ and $\boldsymbol{\beta}_{\mathbf{2}}$.

Then it can be shown that $\bar{f}$ is homotopic to a map $\bar{f}_{1}$ such that $X\left(\bar{f}_{1}\right)$ contains fewer points than $X(\bar{f})$ so that $\bar{f}_{1}^{-1}\left(F_{1}\right)=\bar{f}^{-1}\left(F_{1}\right)$ and $\bar{f}_{1}$ satisfies (1)-(3) above. Thus we may suppose that no such $\beta_{1}$ and $\beta_{2}$ exist. Let $A_{1}, \ldots, A_{n}$ be the closures of the components of $K-\bar{f}^{-1}\left(F_{1}\right)$. After a general position argument, we may assume that the closure of $A_{i} \cap \bar{f}^{-1} \bar{f}^{-1} \bar{f}\left(\operatorname{int}\left(A_{j}\right)\right)$ is the union of a collection of disjoint simple arcs and loops for all $i, j$ such that $1 \leqslant i<j \leqslant n$. Suppose there are arcs $\alpha_{1}$ and $\alpha_{2}$ properly embedded in $A_{i}$ and $A_{j}$ respectively such that $\bar{f}\left(\alpha_{1}\right)=\bar{f}\left(\alpha_{2}\right)$ and $\alpha_{1}$ and $\alpha_{2}$ are not spanning arcs of $A_{i}$ and $A_{j}$ respectively. Let $D_{1}$ be the disk on $A_{i}$ cut off by $\alpha_{1}$ and $D_{2}$ be the disk on $A_{j}$ cut off by $\alpha_{2}$. Observe that $\bar{f}\left(D_{1}\right) \cap \bar{f}\left(A_{j}\right)$ is a collection of disjoint simple arcs and loops properly embedded in the embedded disk $\bar{f}\left(D_{1}\right)$. Thus by making appropriate choices for $\alpha_{1}$ and $\alpha_{2}$, we may assume that $\bar{f}\left(D_{1}\right) \cap \bar{f}\left(A_{j}\right)$ contains a collection of simple loops and exactly one arc $\bar{f}\left(\alpha_{1}\right)=\bar{f}\left(\alpha_{2}\right)$. Let $\beta_{1}$ and $\beta_{2}$ be the closures of $\bar{f}\left(\partial D_{1}-\alpha_{1}\right)$ and $\bar{f}\left(\partial D_{2}-\alpha_{2}\right)$ respectively. Then $\beta_{1} \cup \beta_{2}$ bounds a singular disk $\bar{f}\left(D_{1}\right) \cup \bar{f}\left(D_{2}\right)$ in $M$ so $\beta_{1} \cup \beta_{2}$ bounds a disk $D$ on $F_{1}$. It follows from the usual argument that we may suppose that each arc in $D \cap \bar{f}(K)$ properly embedded in $D$ meets both $\beta_{1}$ and $\beta_{2}$. This contradicts our assumption on the minimality of $X(\bar{f})$, so we may assume that each arc in the closure of $A_{i} \cap \bar{f}^{-1} \bar{f}\left(\operatorname{int}\left(A_{j}\right)\right)$ and properly embedded in $A_{i}$ is a spanning arc of $A_{i}$.

If $\bar{f} \mid \bar{f}^{-1}\left(F_{1}\right)$ is an embedding, Theorem 4 is an immediate consequence of

\section{Lemma 5.13 .}

Let $N$ be a regular neighborhood of $\bar{f}(K)$. Let $G=N \cap F_{1}$. Let $\bar{N}$ be the manifold obtained by splitting $N$ along $G$ and $P: \bar{N} \rightarrow N$ the natural identification map. Denote the copies of $G$ in $\partial \bar{N} \cap P^{-1}(G)$ by $G_{1}$ and $G_{2}$ and observe that $G_{1}$ and $G_{2}$ need not be connected. Let $f_{i}$ be $\bar{f} \mid A_{i}$ for $i=1, \ldots, n$ and $\hat{f}_{i}$ : $A_{i} \rightarrow \bar{N}$ be the map induced by $f_{i}$. Suppose $\mu_{1}$ is a simple loop in $G_{1}$. Then we claim that $\mu_{1}$ is freely homotopic in $\hat{N}$ to a loop in $G_{2}$. Since $\bar{f}(K)$ is a deformation retract of $N,\left(\bigcup_{i=1}^{n} \hat{f}_{i}\left(A_{i}\right)\right) \cap G_{1}$ is a deformation retract of $G_{1}$. Thus $\mu_{1}$ is freely homotopic in $G_{1}$ to a loop in $\bigcup_{i=1}^{n} \hat{f}_{i}\left(\partial A_{i}\right)$. If $\mu_{1}$ is freely homotopic 
to a loop $\lambda$ in $\hat{f}_{i}\left(\partial A_{i}\right)$ in $G_{1}, \mu_{1}$ is freely homotopic in $\bar{N}$ to a loop in $G_{2}$ since $\lambda$ is freely homotopic in $\hat{f}_{i}\left(A_{i}\right)$ to a loop in $\hat{f}_{i}\left(\partial A_{i}\right)-\lambda$. Let

$$
X=\bigcup_{1 \leqslant i<j \leqslant m}\left(\hat{f_{i}}\left(\partial A_{i}\right) \cap \hat{f_{j}}\left(\partial A_{j}\right)\right)
$$

Now $\mu_{1}$ is homotopic in $G_{1}$ to a loop $\mu_{2} \subset \bigcup_{i=1}^{n} \hat{f}_{i}\left(\partial A_{i}\right)$ where $\mu_{2}$ is the union of embedded $\operatorname{arcs} \beta_{1}(t), \ldots, \beta_{S}(t)$ for $t$ in $[0,1]$ such that

(1) $\beta_{i} \cap X=\partial \beta_{i}$ for $i=1, \ldots, S$;

(2) $\beta_{i}(1)=\beta_{i+1}(0)$ for $i=1, \ldots, S-1$;

(3) $\beta_{1}(0)=\beta_{S}(1)$.

Note that a point in $X$ lying on $\hat{f}_{i}\left(A_{i}\right)$ determines a spanning arc in $\hat{f}_{i}\left(A_{i}\right)$ that lies in $\hat{f}_{i}\left(\hat{A}_{i}\right) \cap \hat{f}_{j}\left(A_{j}\right)$ where $1 \leqslant j \leqslant S$ and $j \neq i$. Thus if $\beta_{i}$ lies on $\hat{f}_{i}\left(A_{i}\right), \partial \beta_{i}$ determines two spanning arcs $\alpha_{1}$ and $\alpha_{2}$ on $\hat{f}_{i}\left(A_{i}\right)$. These spanning arcs are not necessarily disjoint. Consider the arc on $\hat{f}_{i}\left(A_{i}\right)$ obtained by running along $\alpha_{1}$ from the component of $\partial \hat{f}\left(A_{i}\right)$ not meeting $\beta_{i}$ to the component of $\partial \hat{f}\left(A_{i}\right)$ meeting $\beta_{i}$, along $\beta_{i}$ and then along $\alpha_{2}$ to the component of $\partial \hat{f}_{i}\left(A_{i}\right)$ not containing $\beta_{i}$. This arc is homotopic rel its endpoints to an $\operatorname{arc} \beta_{i}^{\prime}$ in $\partial \hat{f}_{i}\left(A_{i}\right)$. Thus for each $\beta_{i}$, there is a natural way to associate a $\beta_{i}^{\prime} \subset G_{2}$ for $i=1, \ldots, S$ with $\beta_{i}$. Furthermore we can fit the homotopies above together in a natural way to form a free homotopy from $\mu_{2}$ to $\bigcup_{i=1}^{S} \beta_{i}^{\prime}$. This completes the proof of our claim.

It is a consequence of our claim above and the theorem in [14] that if $\lambda$ is a simple loop in $G_{1}$, there is an annulus $\bar{A}$ embedded in $\bar{N}$ such that $\bar{A} \cap G_{1}=\lambda$ and $\bar{A} \cap G_{2}=\partial \bar{A}-\lambda$.

Note that each component of $\partial G$ which is nullhomotopic in $M$ bounds a disk on $F_{1}$. Let $G^{*}$ be the submanifold of $F_{1}$ obtained by attaching all such disks to $G$. Now any loop in $G^{*}$ that is nullhomotopic in $F_{1}$ is nullhomotopic in $G^{*}$. Since $\bar{f}^{-1}\left(G^{*}\right)$ contains an essential loop, no component of $G^{*}$ is a disk. If a component $G_{1}^{*}$ of $G^{*}$ is an annulus, $\bar{f}$ carries the components of $\bar{f}^{-1}\left(G_{1}^{*}\right)$ to disjoint loops and as a consequence of Lemma $5.13 \bar{f}$ may be taken to be an embedding. Now Theorem 4 follows as above. If $G^{*}$ is planar and has exactly three boundary components $\bar{f} \mid \bar{f}^{-1}\left(G^{*}\right)$ may be taken to be an embedding. It is a consequence of Lemma 5.13 that $\bar{f}$ could be taken to be an embedding and Theorem 4 would follow as above.

We suppose then that if a component of $G^{*}$ is planar it has at least four boundary components. Let $\lambda_{1}, \ldots, \lambda_{r}$ be a collection of essential simple loops properly embedded in $G$ such that

(1) $\operatorname{Arcs} \beta_{1} \subset \lambda_{i}$ and $\beta_{2} \subset \lambda_{j}$ do not exist where $\beta_{1} \cup \beta_{2}$ bounds a disk on $G^{*}$ for $1 \leqslant i<j \leqslant r$.

(2) $G^{*}-\left(\bigcup_{i=1}^{r} \lambda_{i} \cup \partial G^{*}\right)$ is a collection of open annuli (or a disk if $G^{*}$ is closed). 
(3) $\bigcup_{i=1}^{r} \lambda_{i}$ is a deformation retract of $G^{*}$ if $G^{*}$ is not closed.

(4) $\lambda_{i} \cap \lambda_{j}$ contains at most two points for $1 \leqslant i<j \leqslant r$.

The reader may observe that $\lambda_{i} \cap \lambda_{j}$ for $1 \leqslant i<j \leqslant r$ may be assumed to be a single point if $G^{*}$ is not planar.

Let $\bar{A}_{1}, \ldots, \bar{A}_{r}$ be a collection of annuli properly embedded in $\bar{N}$ such that

(1) $P\left(\bar{A}_{i} \cap G_{1}\right)=\lambda_{i}$ for $i=1, \ldots, r$.

(2) $\bar{A}_{i} \cap G_{2}$ is a loop in $\operatorname{int}\left(G_{2}\right)$ for $i=1, \ldots, r$.

After the usual cutting argument, we may suppose that $\bar{A}_{i} \cap \bar{A}_{j}$ is a collection of disjoint spanning arcs for $1 \leqslant i<j \leqslant r$.

Let $N_{1}$ be a regular neighborhood of $G^{*} \cup \bigcup_{i=1}^{r} P\left(\bar{A}_{i}\right)$. It is not difficult to see that if one splits $N_{1}$ along $F_{1} \cap N_{1}$, one obtains a 3-manifold homeomorphic to $G^{*} \times[0,1]$ since $G^{*}$ is a deformation retract of $N_{1} \cap F_{1}$. Let $G_{1}^{*}$ be a component of $G^{*}$. Then it can be seen that $N_{1}$ is a bundle with base $S^{1}$ and fibre $G_{1}^{*}$. Since $N_{1}$ is an orientable 3-manifold, $\partial N_{1}$ is an orientable 2-manifold. Since $N_{1}$ is a bundle with base $S^{1}$ and fibre $G_{1}^{*}, \partial N_{1}$ is a collection of tori. Since $\partial M$ is not empty, $\partial N_{1}$ is not empty. Let $T_{1}$ be a component of $\partial N_{1}$. We claim $T_{1}$ is incompressible in $M$. Let $\lambda_{1}$ be a simple loop in $T_{1} \cap F_{1}$. Since $\pi_{1}\left(G_{1}^{*}\right) \rightarrow \pi_{1}\left(F_{1}\right)$ is monic, $\lambda_{1}$ is essential in $M$. Let $\lambda_{2}$ be a simple loop on $T_{1}$ that meets each loop in $T_{1} \cap F_{1}$ in a single point and crosses $F_{1}$ at each point in $\lambda_{2} \cap F_{1}$. Then the intersection number of $\lambda_{2}$ and $F_{1}$ can be seen to be the same as the cardinality of $F_{1} \cap \lambda_{2}$. Suppose $[\lambda] \in \pi_{1}\left(T_{1}\right)$ is an element such that $\lambda$ is an inessential loop in $M$. Then $[\lambda]=s_{1}\left[\lambda_{1}\right]+s_{2}\left[\lambda_{2}\right]$. Since the intersection number of $\lambda_{1}$ and $F_{1}$ is not zero and that of $\lambda_{2}$ and $F_{1}$ is zero, $s_{1}=0$. Since $\lambda_{2}$ is not nullhomotopic on $F_{1}$ and $F_{1}$ is incompressible in $M, s_{2}=0$. Thus [ $\lambda]=0 \in \pi_{1}\left(T_{1}\right)$ and $T_{1}$ is incompressible in $M$. If $T_{1}$ is not parallel to a component of $\partial M, T_{1}$ is an essential embedding of a torus in $M$ and Theorem 4 follows.

Otherwise it can be seen that $N_{1}$ is a deformation retract of $M$ and $M$ may be taken to be a bundle with base $S^{1}$ and fibre $F_{1}$. It follows that $G^{*}$ is connected as is the regular neighborhood in $F_{1}$ of $F_{1} \cap \bar{f}(K)$. Denote such a neighborhood by $F$. Then it can be seen that $\pi_{1}(F) \rightarrow \pi_{1}\left(F_{1}\right)$ is an epimorphism.

We claim that $M$ has a finite sheeted cyclic covering $(\tilde{M}, p)$ homeomorphic to $F_{1} \times S^{1}$. If this is true, there is an essential map $f:(A, \partial A) \rightarrow(\tilde{M}, \partial \widetilde{M})$ since $F_{1}$ is not a disk. But then $p f:(A, \partial A) \rightarrow(\widetilde{M}, \partial \widetilde{M})$ is an essential map and Theorem 4 will follow from Theorem 2 .

The proof of Theorem 4 will be complete when we establish the claim above. Let $\kappa$ be the number of loops in $\bar{f}^{-1}\left(F_{1}\right)$. Let $\left(M^{*}, q\right)$ be the $k$-sheeted cyclic covering of $M$ associated with $F_{1}$. Then there is an embedding $\bar{g}_{1}: K \rightarrow$ $M^{*}$ such that $q \bar{g}_{1}=\bar{f}$. Let $\rho^{*}: M^{*} \rightarrow M^{*}$ be a generator of the group of cover- 
ing translations. Now $\rho^{* i} g_{1}(K) \cap \rho^{* j} g_{1}(K)$ is the union of a collection of disjoint simple loops for $0 \leqslant i<j<k$ since the closure of $A_{i} \cap \bar{f}^{-1} \bar{f}\left(\operatorname{int}\left(A_{j}\right)\right)$ is a collection of disjoint simple spanning arcs of $A_{i}$ and simple loops in $A_{i}$ for $1 \leqslant i<$ $j \leqslant \kappa$.

Let $\bar{F}_{1}$ be a component of $p^{-1}\left(F_{1}\right)$. Let $\kappa_{i j}$ be the intersection number of $\bar{F}_{1}$ and any loop in $\rho^{* i} g_{1}(K) \cap \rho^{* j} g_{1}(K)$ that meets $\bar{F}_{1}$. If no loop in $\rho^{* i} g_{1}(K)$ $\cap \rho^{* j} g_{1}(K)$ meets $F_{1}$, let $\kappa_{i j}=1$ for $0 \leqslant i<j<\kappa$. Let $\tilde{\kappa}$ be the least common multiple of the $\kappa_{i j}$ and 2 .

Let $\left(\tilde{M}, p_{1}\right)$ be the $\tilde{\kappa}$-sheeted cyclic covering space of $M^{*}$ associated with $\bar{F}_{1}$. Then we claim there is an embedding $h_{i}: T \rightarrow \widetilde{M}$ such that $p_{1} h_{i}(T)=$ $\rho^{* i} \bar{g}_{1}(K)$ and $\left(h_{i}(T), p \mid h_{i}(T)\right)$ is a $\tilde{\kappa}$-sheeted cyclic covering of $\rho^{* i} \bar{g}_{1}(K)$ for $0 \leqslant$ $i<\kappa$. Clearly we need only show that $p_{1}^{-1} \bar{g}_{1}(K)$ is a torus. Since, for each simple loop $\lambda \subset \bar{g}_{1}^{-1} q^{-1}\left(F_{1}\right), K-\lambda$ is an open annulus, each nonorientable loop on $K$ has odd intersection number with $\lambda$. Thus $p_{1}^{-1} \bar{g}_{1}(K)$ is a torus.

Let $p=q p_{1}$. Then $(\tilde{M}, p)$ is a $\kappa * \widetilde{\kappa}$-sheeted cyclic covering of $M$. Let $\widetilde{F}_{1}$ be a component of $p^{-1}\left(F_{1}\right)$. Note that each simple loop in $h_{i}(T) \cap h_{j}(T)$ meets $F_{1}$ in at most a single point for $0 \leqslant i<j<\kappa$. Split $\widetilde{M}$ along $\widetilde{F}_{1}$ to obtain a 3 manifold $\widetilde{N}$. Let $P: \widetilde{N} \rightarrow \widetilde{M}$ be the natural identification map and $\hat{h}_{i}:(A, \partial A)$ $\rightarrow(\tilde{N}, \partial \widetilde{N})$ the maps induced by the $h_{i}: T \rightarrow \widetilde{M}$ for $0 \leqslant i<\kappa$.

We suppose that for each $i$ where $0<i<k$ there. is a $j<i$ where $0 \leqslant j$ such that $\hat{h}_{i}(A) \cap \hat{h}_{j}(A)$ contains a spanning arc of $\hat{h}_{i}(A)$. This is possible since $\bigcup_{i=0}^{k}\left(h_{i}(T) \cap \widetilde{F}_{1}\right)$ is connected although it may be necessary to reorder the subscripts on the $h_{i}$ for $0 \leqslant i<k$.

We have that $\hat{h}_{0}(A) \cap \hat{h}_{1}(A)$ is a collection of disjoint simple loops and spanning arcs. Using standard techniques, we can find proper embeddings $\hat{h}_{0}^{\prime}: A$ $\rightarrow \widetilde{N}$ and $\hat{h}_{1}^{\prime}: A \rightarrow \widetilde{N}$ such that

(1) $\hat{h}_{j}^{\prime}(\partial A)=\hat{h}_{j}(\partial A)$ for $j=0,1$.

(2) $\hat{h}_{0}^{\prime}(A) \cap \hat{h}_{1}^{\prime}(A)$ is the union of a collection of disjoint simple spanning arcs.

(3) $\hat{h}_{0}^{\prime}(A) \cap \hat{h}_{1}^{\prime}(A) \subset \hat{h}_{0}(A) \cap \hat{h}_{1}(A)$.

Since $\hat{h}_{0}^{\prime}(A)$ and $\hat{h}_{0}(A)$ differ only on the interior of the union of a collection of disjoint disks on $A$ and $\pi_{2}(\widetilde{N})=0, \hat{h}_{0}^{\prime}(A)$ and $\hat{h}_{0}(A)$ are homotopic rel $\hat{h}_{0}(\partial A)$. Similarly $\hat{h}_{1}(A)$ and $\hat{h}_{1}^{\prime}(A)$ are homotopic rel $\hat{h}_{1}(\partial A)$. Thus if $2 \leqslant i<\kappa$ and $\hat{h}_{i}^{\prime}(A)$ is homotopic rel $\partial \hat{h}_{i}^{\prime}(A)$ to $\hat{h}_{i}(A)$ and if $\alpha_{1}$ is a spanning arc of $\hat{h}_{i}^{\prime}(A)$ in $\hat{h}_{i}^{\prime}(A) \cap \hat{h}_{0}^{\prime}(A), \alpha_{1}$ is homotopic rel its endpoints to a spanning arc in $\hat{h}_{i}(A) \cap$ $\hat{h}_{0}(A)$. A similar statement holds for a spanning arc $\hat{h}_{i}^{\prime}(A)$ in $\hat{h}_{i}^{\prime}(A) \cap \hat{h}_{1}^{\prime}(A)$.

Let $N_{1}$ be a regular neighborhood of $h_{0}^{\prime}(A) \cup \hat{h}_{1}^{\prime}(A)$ in $\widetilde{N}$. Let $\bar{G}_{1}$ be a regular neighborhood of $\left(h_{0}(T) \cup h_{1}(T)\right) \cap \widetilde{F}_{1}$ in $\widetilde{F}_{1}$. We may suppose that $P^{-1} \bar{G}_{1}=N_{1} \cap P^{-1}\left(\widetilde{F}_{1}\right)$. Since $\hat{h}_{0}^{\prime}(A) \cap \hat{h}_{1}^{\prime}(A)$ is a collection of disjoint simple spanning $\operatorname{arcs}$ of $\hat{h}_{0}^{\prime}(A)$ and $\hat{h}_{1}^{\prime}(A)$, there is a homeomorphism $\bar{\theta}_{1}: \bar{G}_{1} \times[0,1]$ 
$\rightarrow N_{1}$ such that

(1) $\bar{\theta}_{1}\left(\bar{G}_{1} \times[0,1]\right)=N_{1}$.

(2) $\bar{\theta}_{1}\left(\bar{G}_{1} \times\{0,1\}\right)=P^{-1} \bar{G}_{1}$.

(3) There are simple loops $\mu_{0}$ and $\mu_{1}$ on $\bar{G}_{1}$ such that $\bar{\theta}_{1}\left(\mu_{0} \times[0,1]\right)=$ $\hat{h}_{0}^{\prime}(A)$ and $\bar{\theta}_{1}\left(\mu_{1} \times[0,1]\right)=\hat{h}_{1}^{\prime}(A)$.

(4) $P \bar{\theta}_{1}(x, 0)=P \bar{\theta}_{1}(x, 1)$ for $x \in \bar{G}_{1}$.

Suppose that $\pi_{1}\left(\bar{G}_{1}\right) \rightarrow \pi_{1}\left(\widetilde{F}_{1}\right)$ is not monic. Then there is a disk $D_{1}$ embedded in $P^{-1}\left(\widetilde{F}_{1}\right)$ such that $D_{1} \cap P^{-1}\left(\bar{G}_{1}\right)=\partial D_{1}$. Thus there is a component $a_{1}$ of $\partial \bar{G}_{1}$ such that $\bar{\theta}_{1}\left(a_{1} \times\{j\}\right)=\partial D_{1}$ for $j=0$ or 1 . We suppose $\bar{\theta}_{1}\left(a_{1} \times\{0\}\right)=$ $\partial D_{1}$. Since $\bar{\theta}_{1}\left(a_{1} \times[0,1]\right) \cup D_{1}$ is an embedded disk and $P^{-1} \widetilde{F}_{1}$ is incompressible, $\bar{\theta}_{1}\left(a_{1} \times\{1\}\right)$ bounds a disk $D_{2}$ embedded in $P^{-1}\left(\widetilde{F}_{1}\right)$. Now $D_{2} \cap$ $P^{-1}\left(\bar{G}_{1}\right)=\partial D_{2}$ and $D_{1} \cup D_{2} \cup \bar{\theta}_{1}\left(a_{1} \times[0,1]\right)$ is a 2-sphere $S^{2}$ embedded in $\widetilde{N}$. It is a consequence of Theorem 8.1 in [15] that $\widetilde{N}$ is irreducible. Thus $S^{2}$ bounds a 3-ball $B^{3}$ embedded in $\widetilde{N}$. It follows that $\bar{\theta}_{1}$ can be extended to a homeomorphism of $\left(\bar{G}_{1} \cup P\left(D_{1}\right)\right) \times[0,1]$ onto $N_{1} \cup B^{3}$. Since $\bar{G}_{1}$ has only finitely many boundary components, we may suppose that there is a surface $G_{1}$ embedded in $F_{1}$ such that $G_{1} \supseteq \bar{G}_{1}$ and $\pi_{1}\left(G_{1}\right) \rightarrow \pi_{1}\left(F_{1}\right)$ is monic. Applying the argument given above inductively, we may suppose that there is an embedding $\theta_{1}: G_{1} \times[0,1] \rightarrow \widetilde{N}$ that extends $\bar{\theta}_{1}$ such that

(1) $\theta_{1}\left(G_{1} \times\{0,1\}\right)=P^{-1} G_{1}$.

(2) There are loops $\mu_{0}$ and $\mu_{1}$ on $G_{1}$ such that $\theta_{1}\left(\mu_{0} \times[0,1]\right)=\hat{h}_{0}^{\prime}(A)$ and $\theta_{1}\left(\mu_{1} \times[0,1]\right)=\hat{h}_{0}^{\prime}(A)$.

(3) $P \theta_{1}(x, 0)=P \theta_{1}(x, 1)$ for $x \in G_{1}$.

Note that $\theta_{1}\left(\partial G_{1} \times[0,1]\right)$ is a collection of disjoint incompressible annuli in $\widetilde{N}$.

Suppose that $\mu_{0}, \ldots, \mu_{k}, G_{k}$, and $\theta_{k}$ have been defined as above for $k<\kappa$. After an argument similar to those given above, we may assume that $\hat{h}_{k+1}^{\prime}(A) \cap \theta_{k}\left(\partial G_{k} \times[0,1]\right)$ is a nonempty collection of disjoint simple arcs. Thus $\hat{h}_{k+1}^{\prime}(A) \cap \theta_{k}\left(G_{k} \times[0,1]\right)$ is a nonempty collection of disks properly embedded in $\theta_{k}\left(G_{k} \times[0,1]\right)$.

Note that if $\hat{h}_{i}^{\prime}\left(c_{1}\right)$ is freely homotopic to a component of $\theta_{k}\left(\partial G_{k} \times\{0,1\}\right)$ in $\theta_{k}\left(\partial G_{k} \times\{0,1\}\right)$, the loop $P \hat{h}_{i}^{\prime}\left(c_{1}\right)=h_{i}(T) \cap \widetilde{F}_{1}$ is isotopic in $\widetilde{F}_{1}$ to a loop that fails to meet $h_{j}(T) \cap \widetilde{F}_{1}$ for $j \neq i, 0 \leqslant j \leqslant k$. This implies that there are arcs $\beta_{1}$ and $\beta_{2}$ in $\bar{f}(K) \cap F_{1}$ such that $\beta_{1} \cap \beta_{2}=\partial \beta_{1}=\partial \beta_{2}, \beta_{1} \cup \beta_{2}$ bounds a disk on $F_{1}$ and $\beta_{1}\left(\beta_{2}\right)$ lies in the image of a component of $\bar{f}^{-1}\left(F_{1}\right)$. This contradicts an earlier hypothesis.

We proceed with the assumption that $\hat{h}_{i}^{\prime}\left(c_{1}\right)$ and $\hat{h}_{i}^{\prime}\left(c_{2}\right)$ are not freely homotopic in $\widetilde{F}_{1}$ to loops in $\partial P^{-1} G_{k}$ for $0 \leqslant i \leqslant k$. We may also assume that each disk $D$ in $\hat{h}_{k+1}^{\prime}(A) \cap \theta_{k}\left(G_{k} \times[0,1]\right)$ contains a spanning arc of $h_{k+1}^{\prime}(A)$ and one of the annuli $\hat{h}_{i}^{\prime}(A)$ where $0 \leqslant i \leqslant k$. Suppose $D \cap \hat{h}_{0}^{\prime}(A)$ contains a spanning arc of $\hat{h}_{k+1}^{\prime}(A)$. We show that $D$ is isotopic rel $\partial D \cap \theta_{k}\left(G_{k} \times\{0,1\}\right)$ to a 
product disk i.e., $\beta \times[0,1]$ where $\beta$ is an arc properly embedded in $G_{k}$. We may assume that $D \cap \hat{h}_{0}^{\prime}(A)$ is the union of a collection of simple spanning arcs of $\hat{h}_{0}^{\prime}(A)$. Let $\alpha_{1}$ be a spanning arc in this collection. Let $D_{1}$ be the closure of one component of $D-\alpha_{1}$. We may suppose that $D_{1} \cap \hat{h}_{0}^{\prime}(A)=\alpha_{1}$. Now $\alpha_{1}$ is homotopic rel its endpoints to a spanning $\alpha_{1}^{\prime}$ in $\hat{h}_{0}(A) \cap \hat{h}_{k+1}(A)$ such that $P\left(\partial \alpha_{1}^{\prime}\right)$ is a single point. Thus there is a point $x_{1}$ in $G_{k}$ such that $\theta_{k}\left(\left\{x_{1}\right\} \times\{0,1\}\right)=$ $\partial \alpha_{1}$.

We will show that the arc $\alpha_{1}$ is homotopic rel its endpoints to $\theta_{k}\left(\left\{x_{1}\right\} \times[0,1]\right)$. Let $\phi_{k}: G_{k} \times[0,1] \rightarrow G_{k}$ be defined by $\phi_{k}(x, t)=x$ for $x$ in $G_{k}, t$ in [0,1]. Let $\beta_{0}=D \cap \theta_{k}\left(G_{k} \times\{0\}\right)$ and $\beta_{1}=D \cap \theta_{k}\left(G_{k} \times\{1\}\right)$. Now $\phi_{k} \theta_{k}^{-1}\left(\beta_{0}\right)=\phi_{k} \theta_{k}^{-1}\left(\beta_{1}\right)$ since $P\left(\beta_{0}\right)=P\left(\beta_{1}\right)$ for $P \theta_{k}\left(\left\{x_{1}\right\} \times\{0\}\right)=$ $P \theta_{k}\left(\left\{x_{1}\right\} \times\{1\}\right)$. Thus $\phi_{k} \theta_{k}^{-1}: D \rightarrow G_{k}$ determines a map of either an annulus or a möbius band into $G_{k}$. Since $P \hat{h}_{k+1}^{\prime}(A)$ is orientable, we see that $\phi_{k} \theta_{k}^{-1}: D$ $\rightarrow G_{k}$ determines a map $\epsilon_{k}:(A, \partial A) \rightarrow\left(G_{k}, \partial G_{k}\right)$.

Consider the map $\bar{\epsilon}_{k}: A \rightarrow G_{k}$ induced by $\phi_{k} \theta_{k}^{-1} \mid D_{1}$. If $\bar{\epsilon}_{k *}$ is monic, $\mu_{0}$ is homotopic to a loop in $\partial G_{k}$ which we have shown leads to a contradiction. It follows that $\bar{\epsilon}_{k}\left(c_{1}\right)$ and $\bar{\epsilon}_{k}\left(c_{2}\right)$ are nullhomotopic in $G_{k}$. Thus the loop $\phi_{k} \theta_{k}^{-1}\left(D_{1} \cap \hat{h}_{i}^{\prime}(A)\right)$ is nullhomotopic and the arc $\alpha_{1}$ is homotopic rel its boundary to $\theta_{1}\left(\left\{x_{1}\right\} \times[0,1]\right)$ as was to be shown.

It follows that after a homotopy of $\hat{h}_{k+1}^{\prime}$ rel $\partial A$, we may suppose that $\theta_{k}^{-1}\left(\hat{h}_{k+1}^{\prime}(A)\right)$ is a collection of product disks i.e. $\phi_{k} \theta_{k}^{-1}\left(\hat{h}_{k+1}^{\prime}(A)\right)$ is a collection of arcs. But now $\theta_{k}$ can be extended in an obvious way to $\theta_{k+1}: G_{k+1} \times$ $[0,1] \rightarrow \widetilde{N}$ where $\widetilde{F}_{1} \supseteq G_{k+1} \supseteq G_{k}$ so that

(1) $\theta_{k+1}\left(G_{k+1} \times\{0,1\}\right)=P^{-1}\left(G_{k+1}\right)$.

(2) There are loops $\mu_{0}, \ldots, \mu_{k+1}$ on $G_{k+1}$ such that $\theta_{k+1}\left(\mu_{i} \times[0,1]\right)$ $=\hat{h}_{i}^{\prime}(A)$ for $i=1, \ldots, k+1$.

(3) $P \theta_{k+1}(x, 0)=P \theta_{k+1}(x, 1)$ for $x \in G_{k+1}$.

(4) $\pi_{1}\left(G_{k+1}\right) \rightarrow \pi_{1}\left(\widetilde{F}_{1}\right)$ is monic.

It follows that there is an embedding $\theta_{\kappa}: G_{\kappa} \times[0,1] \rightarrow \widetilde{N}$ such that

(1) $P \theta_{\kappa}(x, 0)=P \theta_{\kappa}(x, 1)$ for $x \in G_{\kappa}$.

(2) $P \theta_{k}\left(G_{k} \times\{0\}\right) \supseteq \bigcup_{i=0}^{k-1} h_{i}(T) \cap \widetilde{F}_{1}$.

(3) $\theta_{\kappa *}$ is monic.

Since $\theta_{\kappa}\left(G_{\kappa} \times\{0,1\}\right) \supseteq \bigcup_{i=0}^{\kappa-1} \hat{h}_{i}^{\prime}(\partial A), \theta_{\kappa *}$ is onto. Thus $P \theta_{\kappa}\left(G_{\kappa} \times\{0\}\right.$ is a deformation retraction of $\widetilde{F}_{1}$. But it can now be seen that $P \theta_{\kappa}\left(G_{\kappa} \times[0,1]\right)$ is homeomorphic to $G_{\kappa} \times S^{1}$ and is a deformation retraction of $\widetilde{M}$. It follows that $\widetilde{M}$ is homeomorphic to $G_{\kappa} \times S^{1}$ and Theorem 4 follows.

VII. Other theorems and applications.

THEOREM 5. Let $M$ be a compact, bounded, irreducible orientable 3-manifold. If $M$ admits an S-essential map, $M$ admits an essential embedding $h: T \rightarrow M$. 
Proof. Let $f: T \rightarrow M$ be an $S$-essential map. By Theorem 4, $M$ admits an essential embedding $g_{1}:(A, \partial A) \rightarrow(M, \partial M)$ or an essential embedding $h: T \rightarrow$ $M$. Assume the former and that $f$ is not homotopic to a map $f_{1}$ such that $f_{1}(T)$ $\cap g_{1}(A)$ is empty. It is a consequence of Lemma 5.6 that there is an embedding $h: T \rightarrow M$ such that $h^{-1}(T)$ contains a simple loop $\lambda$ so that $h(\lambda)$ is freely homotopic in $g_{1}(A)$ to a loop in $\partial g_{1}(A)$ and $h_{*}$ is monic. We may suppose that $\lambda_{1}$ is a simple essential loop in $f^{-1}\left(g_{1}(A)\right)$. Since $\lambda_{1}$ is essential and $f$ is $S$-essential $f\left(\lambda_{1}\right)$ is not freely homotopic to a loop in $\partial M$. Since $f\left(\lambda_{1}\right)$ is freely homotopic to a loop in $g_{1}(\partial A), f\left(\lambda_{1}\right)$ is freely homotopic to a multiple of $h(\lambda)$ and $h(\lambda)$ is not freely homotopic to a loop in $\partial M$. Thus $h$ is essential.

If $f$ is homotopic to a map $f_{1}$ such that $f_{1}(T) \cap g_{1}(A)$ is empty, we trade $f$ for $f_{1}$ and assume $f(T) \cap g_{1}(A)$ is empty. Let $M_{1}$ be the manifold obtained by removing the interior of a regular neighborhood of $g_{1}(A)$ from $M$. If $f: T \rightarrow M_{1}$ is essential, we repeat the argument above. As a result of this argument, either we find an essential embedding $g_{2}:(A, \partial A) \rightarrow\left(M_{1}, \partial M_{1}\right)$ or an embedding $h: T \rightarrow$ $M_{1}$ such that $h: T \rightarrow M$ is essential. In case we find $g_{2}: A \rightarrow M_{1}$ and $f$ is homotopic to a map $f_{1}$ such that $f_{1}(T) \cap g_{2}(A)$ is empty, we suppose that $f(T) \cap g_{2}(A)$ is empty and let $M_{2}$ be the 3-manifold obtained from $M_{1}$ by removing the interior of a regular neighborhood of $g_{2}(A)$ from $M_{1}$. If $f$ is not homotopic to a map such that $f(T) \cap g_{2}(A)$ is empty, we find a map $h: T \rightarrow M_{1}$ as above so that $h: T \rightarrow M$ is essential.

We continue the process above until either we have found an essential map $h: T \rightarrow M$ or $f: T \rightarrow M_{i} \subset M$ is inessential in $M_{i}$. Note that we have constructed a partial hierarchy for $M$ and the process above must terminate.

Suppose $\partial M_{i}$ is not incompressible in $M_{i}$. Then there is a disk $D$ properly embedded in $M_{i}$ such that $\partial D$ is essential in $\partial M_{i}$. We may suppose that $f^{-1}(D)$ is the union of a collection of simple loops. If $\lambda$ in $f^{-1}(D), \lambda$ is nullhomotopic on $T$ since $f_{*}$ is monic. Thus after the usual argument we may suppose that $f(T)$ $\cap D$ is empty. It can now be seen that we can assume that $\partial M_{i}$ is incompressible and $M_{i}$ is irreducible. It follows from standard arguments that $f$ is homotopic to a map into $\partial M_{i}$. Suppose that $f_{1}: T \rightarrow \partial M_{i}$ is homotopic to $f$. Let $T_{1}=f_{1}(T)$.

Note that $f_{1}: T \rightarrow T_{1}$ is homotopic to a covering map by Lemma 1.4.3 in [15] since $f_{1 *}: \pi_{1}(T) \rightarrow \pi_{1}\left(T_{1}\right)$ is monic and that $T_{1}$ is a torus. Let $h: T \rightarrow$ $T_{1}$ be an embedding. Then $h_{*}: \pi_{1}(T) \rightarrow \pi_{1}(M)$ is monic since $\partial M_{i}$ is incompressible in $M_{i}$ (and in $M$ ). It can now be seen that $h$ is an essential map. This completes the proof of Theorem 5 .

THEOREM 6. Let $M$ be a compact, orientable, irreducible, boundary irreducible 3-manifold. Let the torus $T_{1}$ be a component of $\partial M$. Suppose $M$ admits an essential embedding $g:(A, \partial A) \rightarrow\left(M, T_{1}\right)$. If $g(A)$ separates $M$, we denote 
the closures of the components of $M-g(A)$ by $M_{1}$ and $M_{2}$. Then either $M$ admits an essential embedding of $T$ or $g(A)$ separates $M$ and $M_{j}$ is either a solid torus or homeomorphic to $T \times[0,1]$ for $j=1,2$.

Proof. Let $A_{1}$ and $A_{2}$ be the closures of the components of $T_{1}-g(\partial A)$. Let $g_{j}: T \rightarrow M$ be an embedding such that $g_{j}(T)$ is parallel to the torus $A_{j} \cup g(A)$ for $j=1,2$. If $g_{1}$ is not essential, either $g_{1 *}: \pi_{1}(T) \rightarrow \pi_{1}(M)$ is not monic or $g_{1}(T)$ is parallel to a component of $\partial M$. In the former case, the usual argument shows $g_{1}(T)$ bounds a solid torus $N$ in $M$. Since $T_{1}$ is incompressible in $M, T_{1}$ is not contained in $N$. Thus $g(A)$ is not contained in $N$. This shows that $M_{1}$ may be taken to be a solid torus.

In the latter case $g_{1}(T)$ and a component $F$ of $\partial M$ bound a submanifold $N$ of $M$ homeomorphic to $T \times[0,1]$. Since $g$ is essential, $F \neq T_{1}$ and $g(A)$ is not contained in $N$. Since $g_{1}(T)$ is parallel to $A_{1} \cup g(A)$, the desired manifold $M_{1}$ is the union of $N$ with the submanifold of $M$ bounded by $g_{1}(T)$ and $A_{1} \cup g(A)$. We observe that $\partial M_{1}=\left(\partial N-g_{1}(T)\right) \cup A_{1} \cup g(A)$.

A similar argument establishes the existence of the desired $M_{2}$. This completes the proof of Theorem 6 .

Corollary to Theorem 6. Let $M$ be the knot space of a knot $K$. If $M$ admits an essential map of a torus but not an essential embedding of a torus, $K$ is a torus knot.

Proof. This result follows immediately from Theorem 4 and Theorem 6. We observe that Theorem 7 below gives a partial answer to question $T$ on p. 101 in [8]. Theorem 7 also seems to be related to problem 4, p. 168 in [7]. This question has been partially answered in [4].

THEOREM 7. Let $M$ be the space of a knot K. Suppose $\pi_{1}(M)$ has a subgroup $A$ isomorphic to $Z \oplus Z$ such that $A$ is not conjugate to a subgroup of a peripheral subgroup of $\pi_{1}(M)$. Then either $K$ is a torus knot or $M$ admits an essential embedding $g: T \rightarrow M$ such that $g_{*} \pi_{1}(T)$ is not conjugate to a subgroup of a peripheral subgroup of $\pi_{1}(M)$.

Proof. Let $f: T \rightarrow M$ be a map such that $f_{*} \pi_{1}(T)=A \subseteq \pi_{1}(M)$. Note that $f$ is essential since if $f$ were homotopic to a map into $\partial M, A$ would be conjugate to a subgroup of a peripheral subgroup of $\pi_{1}(M)$. Thus by Theorem 6 either $K$ is a torus knot or $M$ admits an essential embedding $g: T \rightarrow M$. But since $g(T)$ is not parallel to $\partial M, g_{*} \pi_{1}(T)$ is not conjugate to a subgroup of $\pi_{1}(\partial M) \subseteq$ $\pi_{1}(M)$. This completes the proof of Theorem 7 .

In Theorem 8 below we give a positive answer to question 3 on p. 24 in [9] in case $M$ is a bounded, irreducible, orientable 3-manifold and the surface in question has genus one. An example in [13] shows that Theorem 8 does not hold if 
either $M$ is not required to be bounded or $M$ is not irreducible.

THEOREM 8. Let $M$ be a compact, bounded, irreducible, orientable 3manifold. Let $f: T \rightarrow M$ be a map such that $f_{*}: \pi_{1}(T) \rightarrow \pi_{1}(M)$ is monic. Then there exists an embedding $g: T \rightarrow M$ such that $g_{*}: \pi_{1}(T) \rightarrow \pi_{1}(M)$ is monic.

Proof. Let $D$ be a disk properly embedded in $M$. After a homotopy we may suppose that $f^{-1}(D)$ is the union of a collection of disjoint simple loops. Since $f_{*}$ is monic, each loop in $f^{-1}(D)$ is inessential on $T$. Thus we may suppose that $f^{-1}(D)$ is empty and $\partial M$ is incompressible.

If $f$ is $S$-essential Theorem 8 is a consequence of Theorem 5 .

If $f$ is homotopic to a map into $\partial M$, one component of $\partial M$ is an incompressible torus embedded in $M$ and Theorem 8 follows.

Thus we may suppose that $f$ is $W$-essential. It is a consequence of Theorem 4 that $M$ admits an essential embedding of either a torus or an annulus. In case of the former, Theorem 8 follows. Otherwise we assume that $f$ is in general position with respect to an essential annulus $A_{1}$ in $M$. If $f$ is not homotopic to a map $f_{1}$ such that $f_{1}(T) \cap A_{1}$ is empty Theorem 8 is a consequence of Lemma 5.6. Otherwise we assume that $f(T) \cap A_{1}$ is empty and remove the interior of a regular neighborhood of $A_{1}$ from $M$ to obtain a 3-manifold $M_{1}$.

If $f: T \rightarrow M_{1}$ is homotopic to a map into $\partial M_{1}$, Theorem 8 follows as above. Otherwise we repeat the argument above to obtain an essential embedding $A_{2}$ of an annulus in $M_{1}$. It is a consequence of the theorem on p. 60 in [15] that the construction above can be carried out at most finitely many times. Theorem 8 follows.

THEOREM 9. Let $M$ and $N$ be compact, connected, bounded, irreducible 3-manifolds. Let $\Phi: \pi_{1}(M) \rightarrow \pi_{1}(N)$ be a monomorphism. Suppose that $\partial M$ is an incompressible torus and that $M$ admits no essential embedding of an annulus. Then there is a submanifold $N_{1}$ of $N$ and a covering map $\psi: M \rightarrow N_{1}$ such that $\psi_{*}=\Phi: \pi_{1}(M) \rightarrow \pi_{1}(N)$.

Remark. The following example shows that Theorem 9 is false if $M$ is allowed to admit essential embeddings of annuli: Let $p, q$ and $r$ be integers and $p$ and $q r$ relatively prime. Let $M$ be the space of a $p, q$ torus knot and $N$ the space of a $p$, qr torus knot. Then $\pi_{1}(M)=\left\langle x, y: x^{p}=y^{q}\right\rangle$ and $\pi_{1}(N)=$ $\left\langle w, z: w^{p}=z^{q r}\right\rangle$. We define a monomorphism $\Phi: \pi_{1}(M) \rightarrow \pi_{1}(N)$ by setting $\Phi(x)=w$ and $\Phi(y)=z^{r}$ and extending in the natural way. We observe that $\Phi\left(\pi_{1}(M)\right)$ is not of finite index in $\pi_{1}(N)$ since $\left\langle x, y: x^{p}=y^{q}, y=1\right\rangle$ is a finite group while $\left\langle w, z: w^{p}=z^{q r}, z^{r}=1\right\rangle$ is isomorphic to the free product of the integers $\bmod p$ with integers $\bmod r$ which is not finite. Thus since $M$ is compact, $M$ is not a finite sheeted covering of $N$. Schubert has shown in [10] that a torus 
knot has no companions. Thus if Theorem 9 held, $M$ would be a finite sheeted covering space of $N$. This is impossible.

Proof of Theorem 9. Using standard techniques, we can find a map $f: M \rightarrow N$ such that $f_{*}=\Phi$ since $\pi_{2}(N)=0$. Suppose $D$ is a disk properly embedded in $N$ such that $\partial D$ is essential in $\partial N$. Then $f$ is homotopic to a map $f_{1}$ such that $f_{1}^{-1}(D)$ is the union of a (possibly empty) collection of disjoint disks properly embedded in $M$ by Lemma 1.1 in [3]. Since $\partial M$ is incompressible and $\pi_{2}(M)=0$, we may assume that $f_{1}^{-1}(D)$ is empty. We replace $f$ by $f_{1}$. Thus we may suppose that $f(M)$ lies in the complement of the interior of a regular neighborhood of $D$. It follows that we may suppose that $\partial N$ is incompressible.

Suppose $A$ is an essential annulus properly embedded in $N$. As above we may assume that $f^{-1}(A)$ is a collection of disjoint annuli properly embedded in $M$. Since $M$ admits no essential annuli, these annuli are parallel to annuli in $\partial M$. It follows that we may suppose $f^{-1}(A)$ is empty and thus that $N$ admits no essential embeddings of annuli.

If $f: \partial M \rightarrow N$ is homotopic to a map into $\partial N$, we may suppose that $f(\partial M) \subset \partial N$. But then by Theorem 6.1 in [15], $f$ is homotopic to a covering map since $M$ is not homeomorphic to $T \times[0,1]$. Since this would establish Theorem 9, we may assume that $f: \partial M \rightarrow N$ is an essential map. By Theorem 4, $N$ admits an essential embedding $g_{1}: T \rightarrow N$ or $h:(A, \partial A) \rightarrow(N, \partial N)$. We have assumed that such an $h$ does not exist. Let $g_{i}: T \rightarrow N$ for $i=1, \ldots, n$ be a maximal collection of essential embeddings such that

(1) $g_{i}(T) \cap g_{j}(T)$ is empty for $1 \leqslant i<j \leqslant n$.

(2) $g_{i}(T)$ and $g_{j}(T)$ are not parallel for $1 \leqslant i<j \leqslant n$.

The hierarchy theorem guarantees the finiteness of this collection.

We assume that $f: M \rightarrow N$ is transverse with respect to $\bigcup_{i=1}^{n} g_{i}(T)$. It is a consequence of Lemma 1.1 in [3] that we may take $f^{-1}\left(\bigcup_{i=1}^{n} g_{i}(T)\right)=F$ to be an incompressible surface. It can be seen as above that we may suppose no component of $F$ is an annulus. Thus $F$ is the union of a collection of tori.

Suppose $T_{1}$ is a component of $F$ and $T_{1}$ is parallel to $\partial M$. Then $f$ is homotopic to a map $f_{1}:(M, \partial M) \rightarrow\left(N, f\left(T_{1}\right)\right)$ so that $f_{1}^{-1} f\left(T_{1}\right)$ is the union of a nonempty collection of disjoint incompressible tori. We let $f=f_{1}$ without loss of generality.

If $f^{-1} f(\partial M)=\partial M$, we apply Theorem 6.1 in [15] as above to complete the proof of Theorem 9 . Note that the torus $f(\partial M)$ separates $N$ since it represents a boundary of a chain in $C_{3}\left(N ; Z_{2}\right)$. Suppose $f^{-1} f(\partial M)$ is not connected. Let $N_{1}$ be the closure of a component of $N-f(\partial M)$ that contains a component of $\partial N$. Let $M_{1}$ be a component of $f^{-1}\left(N_{1}\right)$. Now $f \mid M_{1}:\left(M_{1}, \partial M_{1}\right) \rightarrow\left(N_{1}, \partial N_{1}\right)$. Since $f\left(\partial M_{1}\right)$ does not meet $\partial N$, there is no homotopy $f_{t}:\left(M_{1}, \partial M_{1}\right) \rightarrow$ $\left(N_{1}, \partial N_{1}\right)$ such that $f_{0}=f \mid M_{1}$ and $f_{1}$ is a covering map. Thus by Theorem 6 
in [15], there is a homotopy as above such that $f_{1}\left(M_{1}\right) \subseteq \partial N_{1}$. It follows from standard arguments that we may suppose that $f^{-1} f(\partial M)=\partial M$ and Theorem 9 follows.

We suppose that $f(\partial M) \cap \bigcup_{i=1}^{n} g_{i}(T)$ is empty and $f: \partial M \rightarrow N$ is not homotopic to a map into $g_{i}(T)$ where $1 \leqslant i \leqslant n$ and show this leads to a contradiction. This will complete the proof of Theorem 9. It is a consequence of Lemma 5.4 that $g_{i}: T \rightarrow N$ is an $S$-essential map for $1 \leqslant i \leqslant n$ since it has been supposed that $N$ does not admit an essential map of an annulus. Let $N_{2}$ be a regular neighborhood of $\bigcup_{i=1}^{n} g_{i}(T)$ in $N$ and $N_{3}=N-\operatorname{int}\left(N_{2}\right)$. Let $N_{1}$ be the component of $N_{3}$ that contains $f(\partial M)$. By assumption $f: \partial M \rightarrow N_{1}$ is an essential map. Thus by Theorem 4 there is an essential embedding $g_{n+1}: T \rightarrow N_{1}$ or an essential embedding $h:(A, \partial A) \rightarrow\left(N_{1}, \partial N_{1}\right)$. Since $g_{i}, i=1, \ldots, n$, is a maximal collection of essential embeddings $g_{n+1}$ does not exist. We show below that it is impossible for $h$ to exist.

Note that $h(\partial A)$ does not lie on $\partial N$ since $N$ admits no essential embeddings of annuli. If $h$ carries one component of $\partial A$ to $\partial N$ and the other to $\partial N_{1}-\partial N$, one of the $g_{i}$ is not $S$-essential so we may suppose that $h(\partial A) \subset \partial N_{1}-\partial N$.

If $h(\partial A)$ is contained in a single component of $\partial N_{1}-\partial N$, it is a consequence of Theorem 6 that a representative of the generator of $\pi_{1}(h(A))$ is freely homotopic to a loop in $\partial N$. Thus an essential loop on $\partial N_{1}-\partial N$ is freely homotopic to a loop in $\partial N$. This is impossible since $g_{i}$ is $S$-essential for $i=1, \ldots, n$.

We suppose that $h\left(c_{1}\right)$ and $h\left(c_{2}\right)$ lie on distinct components of $\partial N_{1}-\partial N$. We denote these components by $T_{1}$ and $T_{2}$ respectively. Suppose that $f$ is homotopic to a map $f_{1}$ such that $f_{1}(T) \cap h(A)$ is empty. Let $\hat{N}_{2}$ be a regular neighborhood of $T_{1} \cup T_{2} \cup g(A)$ in $N_{1}$. Let $\hat{N}_{1}$ be the closure of $N_{1}-\hat{N}_{2}$. Now $\hat{N}_{1} \cap \hat{N}_{2}$ is a torus $T_{0}$. Suppose $T_{0}$ is not essential in $N_{1}$. If $\pi_{1}\left(T_{0}\right) \rightarrow \pi_{1}(N)$ is not monic, the usual argument shows that $T_{0}$ bounds a solid torus $\hat{N}_{0} \subseteq N_{1}$. But $\hat{N}_{0}$ does not contain $T_{1}$ so $\hat{N}_{0}=\hat{N}_{1}$. But then $f_{*}: \pi_{1}(\partial M) \rightarrow \pi_{1}\left(\hat{N}_{1}\right)$ is not monic which is impossible. If $T_{0}$ is parallel to a component of $\partial N_{1}, T_{0}$ is parallel to a component of $\partial N_{1}-\left(T_{1} \cup T_{2}\right)$ so $\hat{N}_{1}$ is homeomorphic to $T \times$ $[0,1]$. This is impossible since $f: \partial M \rightarrow N_{1}$ is not homotopic to a map into $\partial N_{1}$.

Thus we may assume that $f: \partial M \rightarrow N_{1}$ is not homotopic to a map $f_{1}: \partial M$ $\rightarrow N_{1}$ such that $f_{1}(\partial M) \cap h(A)$ is empty.

After the usual argument, we may assume that $\partial M \cap f^{-1} h(A)$ is the union of a collection of disjoint, simple, essential loops $\lambda_{1}, \ldots, \lambda_{m}$. It can be seen that $f: \partial M \rightarrow N_{1}$ is homotopic to a map $f_{1}: \partial M \rightarrow N_{1}$ such that $f_{1}^{-1} h(A)=$ $f_{1}^{-1} h\left(c_{1}\right)=f^{-1} h(A)$. Now the restriction of $f_{1}$ to the closures of the components of $\partial M-\bigcup_{i=1}^{m} \lambda_{i}$ determines a collection of maps $\hat{f}_{i}:(A, \partial A) \rightarrow\left(N_{1}, T_{1}\right)$ for $i=1, \ldots, m$ and it can be seen that if $\hat{f}_{i}$ is not essential for some $i$ where 
$1 \leqslant i \leqslant m, f_{1}: \partial M \rightarrow N_{1}$ is not essential for it will be homotopic to a map into $T_{1}$. Thus by Theorem 2 there is an essential embedding $h_{1}:(A, \partial A) \rightarrow\left(N_{1}, T_{1}\right)$.

It has been shown above that if $h(\partial A)$ is contained in a single component of $\partial N_{1}-\partial N, N_{1}$ admits an essential embedding of $T$. This is a contradiction. Thus $f: \partial M \rightarrow N_{1}$ is homotopic to a map $f_{1}: \partial M \rightarrow \partial N_{1}$. This completes the proof of Theorem 9 .

THEOREM 10. Let $M$ and $N$ be compact connected, irreducible, boundary irreducible 3-manifolds. Suppose that $\partial M$ is a torus and that $M$ does not admit an essential embedding of an annulus. If $\Phi: \pi_{1}(M) \rightarrow \pi_{1}(N)$ is an isomorphism, there is a homeomorphism $h$ of $M$ onto $N$ such that $h_{*}=\Phi$.

Proof. By Theorem 9 there is a submanifold $N_{1}$ of $N$ and covering map $\psi: M \rightarrow N_{1}$ such that $\hat{\psi}_{*}=\Phi: \pi_{1}(M) \rightarrow \pi_{1}(N)$. Thus $\hat{\psi}_{*}$ is an isomorphism so $\pi_{1}\left(N_{1}\right) \rightarrow \pi_{1}(N)$ and $\psi_{*}: \pi_{1}(M) \rightarrow \pi_{1}\left(N_{1}\right)$ are isomorphisms and $\psi$ is an embedding. Let $N_{2}=\operatorname{cl}\left(N-N_{1}\right)$. It is a consequence of van Kampen's theorem that $\pi_{1}(N)$ is the free product of $\pi_{1}\left(N_{1}\right)$ and $\pi_{1}\left(N_{2}\right)$ with amalgamation over $\pi_{1}\left(\partial N_{1}\right)$ since $\pi_{1}\left(\partial N_{1}\right) \rightarrow \pi_{1}(N)$ is monic. It is a consequence of Lemma 4.2 in [2] that $\pi_{1}\left(\partial N_{1}\right) \rightarrow \pi_{1}\left(N_{2}\right)$ is an epimorphism. It follows from Lemma 1.1 in [3] that $N_{2}$ is homeomorphic to $T \times[0,1]$. Theorem 10 follows.

REMARK. Let $M$ be the space of the square knot and $N$ the space of the granny. Then it is known that $\pi_{1}(M) \cong \pi_{1}(N)$ but that $M$ is not homeomorphic to $N$ so the requirement in Theorem 10 that $M$ does not admit an essential embedding of an annulus cannot be omitted.

\section{BIBLIOGRAPHY}

1. E. M. Brown, Unknotting in $M^{2} \times I$, Trans. Amer. Math. Soc. 123 (1966), 480505. MR 33 \#6640.

2. E. M. Brown and R. H. Crowell, The augmentation subgroup of a link, J. Math. Mech. 15 (1966), 1065-1074. MR 33 \#4920.

3. C. D. Feustel, Some applications of Waldhausen's results on irreducible surfaces, Trans. Amer. Math. Soc. 149 (1970), 575-583. MR 41 \#6188.

4. C. D. Feustel and N. Max, On a problem of R. H. Fox, Topology of Manifolds (Proc. Inst. Univ. of Georgia, Athens, Ga., 1969), Markham, Chicago, Ill., 1970, pp. 132139. MR 42 \#8472.

5. C. D. Feustel, On embedding essential annuli in $M^{3}$, Canad. J. Math. 26 (1974), $1341-1350$.

6. James W. Cannon and C. D. Feustel, Essential embeddings of annuli and Mobius bands in 3-manifolds, Trans. Amer. Math. Soc. 215 (1976), 219-239.

7. R. H. Fox, Some problems in knot theory, Topology of 3-Manifolds and Related Topics (Proc. Univ. of Georgia Inst., 1961), Prentice-Hall, Englewood Cliffs, N. J., 1962, pp. 168-176. MR 25 \#3523.

8. L. Neuwirth, Knot groups, Ann. of Math. Studies, no. 56, Princeton Univ. Press, Princeton, N. J., 1965. MR 31 \#734.

9. C. D. Papakyriakopoulos, On Dehn's lemma and the asphericity of knots, Ann. of Math. (2) 66 (1957), 1-26. MR 19, 761.

10. H. Schubert, Knoten und Vollringe, Acta Math. 90 (1953), 131-286. MR 17, 291. 
11. A. Shapiro and J. H. C. Whitehead, A proof and extension of Dehn's lemma, Bull. Amer. Math. Soc. 64 (1958), 174-178. MR 21 \#2242.

12. J. Stallings, On the loop theorem, Ann. of Math. (2) 72 (1960), 12-19. MR. 22 \#12526.

13. F. Waldhausen, Grupen mit Zentrum und 3-dimensionale Mannigfaltigkeiten, Topology 6 (1967), 505-517. MR 38 \#5223.

14. - Eine Verallgemeinerung des Schleifensatzes, Topology 6 (1967), 501504. MR 36 \#3366.

15. On irreducible 3-manifolds which are sufficiently large, Ann. of Math. (2) 87 (1968), 56-88. MR 36 \#7146.

16. The word problem in fundamental groups of sufficiently large irreducible 3-manifolds, Ann. of Math. (2) 88 (1968), 272-280. MR 39 \#2167.

17. On the determination of some bounded 3-manifolds by their fundamental groups alone, Proc. Internat. Sympos. on Topology and its Applications, Herieg-Novi, Yugoslavia, Aug. 25-31, 1968, Beograd, 1969, pp. 331-332.

18. J. H. C. Whitehead, On 2-spheres in 3-manifolds, Bull. Amer. Math. Soc. 64 (1958), 161-166. MR 21 \#2241.

DEPARTMENT OF MATHEMATICS, VIRGINIA POLYTECHNIC INSTITUTE AND STATE UNIVERSITY, BLACKSBURG, VIRGINIA 24061 